\title{
1 Multifractality in postural sway supports quiet eye training in 2 aiming tasks: A study of golf putting
}

4 Noah Jacobson ${ }^{1}$, Quinn Berleman-Paul ${ }^{1}$, Madhur Mangalam², Damian G. Kelty-Stephen ${ }^{1}$, and 5 Christopher Ralston ${ }^{1}$

$7 \quad{ }^{1}$ Department of Psychology, Grinnell College, Grinnell, IA 50112, USA

$8{ }^{2}$ Department of Physical Therapy, Movement and Rehabilitation Sciences, Northeastern University, 9 Boston, MA 02115, USA

11 Author for correspondence:

12 Damian G. Kelty-Stephen

13 e-mail: keltysda@ grinnell.edu

Ethics statement. All participants provided verbal and written informed consent approved by the Institutional Review Board (IRB) at Grinnell College (Grinnell, IA).

17 Data accessibility. All data analyzed in the present study are available upon request.

18 Author contributions: Noah Jacobson: Conceptualization, Methodology, Software, Investigation, Resources, Data curation, Writing - original draft, Writing - review \& editing. Quinn Berleman-Paul: Methodology, Software, Investigation, Resources, Data curation, Writing - review \& editing. Madhur Mangalam: Conceptualization, Methodology, Software, Validation, Formal analysis, Data curation, Writing - review \& editing, Visualization, Supervision, Project administration. Damian G. KeltyStephen: Conceptualization, Methodology, Validation, Formal analysis, Writing - original draft, Writng - review \& editing, Supervision. Christopher Ralston: Conceptualization, Methodology,

25 Validation, Resources, Supervision, Project administration, and Funding Acquisition.

26 Competing interests. The authors have no competing interests to declare.

\section{Supplementary materials}

29 Supplementary Table S1. Subset of Poisson regression model output including main and interaction 


\section{Abstract}

32 The 'quiet eye' (QE) approach to visually-guided aiming behavior invests fully in perceptual 33 information's potential to organize coordinated action. Sports psychologists refer to QE as the stillness 34 of the eyes during aiming tasks and increasingly into self- and externally-paced tasks. Amidst the 35 'noisy' fluctuations of the athlete's body, quiet eyes might leave fewer saccadic interruptions to the 36 coupling between postural sway and optic flow. Postural sway exhibits fluctuations whose multifractal 37 structure serves as a robust predictor of visual and haptic perceptual responses. Postural sway generates 38 optic flow centered on an individual's eye height. We predicted that perturbing the eye height by 39 attaching wooden blocks below the feet would perturb the putting more so in QE-trained participants 40 than participants trained technically. We also predicted that QE's efficacy and responses to

41 perturbation would depend on multifractality in postural sway. Specifically, we predicted that less 42 multifractality would predict more adaptive responses to the perturbation and higher putting accuracy. 43 Results showed that lower multifractality led to more accurate putts, and the perturbation of eye height 44 led to less accurate putts, particularly for QE-trained participants. Models of radial error (i.e., the 45 distance between the ball's final position and the hole) indicated that lower estimates of multifractality 46 due to nonlinearity coincided with a more adaptive response to the perturbation. These results suggest 47 that reduced multifractality may act in a context-sensitive manner to restrain motoric degrees of 48 freedom to achieve the task goal.

49 Keywords: biotensegrity, degree of freedom, fractal, posture, tensegrity, visual adaptation 


\section{Introduction}

51

52

53

54

55

56

57

58

59

60

61

62

63

64

65

66

67

68

69

70

71

72

73

74

75

76

77

78

79

80

81

82

83

84

85

86

87

88

89

90

91

92

93

94

95

96

Aiming our behaviors into the visible world requires an ongoing awareness of how our body fits in the world around it. The difficulty of sorting out this bodily fit finds its classic encoding in the "degrees of freedom' problem: even if a fine-grained choreography of goal-directed movement that stipulates all individual motoric degrees of freedom were possible, this fine-grained choreography would probably be too rigid to adapt to the task or context (Bernstein, 1967). However, another perspective suggests that the abundance of degrees of freedom is not a problem but a 'blessing' (Latash, 2012). According to this view, there is no need to stipulate the infinitude of motoric variables at very fine grains, and on the contrary, task or intention organizes the movement system at the relatively coarse grain of the whole body and context (Latash, 2020). The infinitude thus becomes a guarantee of adaptive flexibility. This positive view of motor abundance appears in recent studies of aiming behaviors like the golf swing, whereby a clearly defined target in the context draws visual attention away from specific postures of the body, and indeed, experts with their eyes on the target show no single optimized mode of execution (Morrison et al., 2016). Hence, instead of requiring explicit choreography, the movement system's organization may readily emerge from perceptual linkages to the surrounding context (Stoffregen, 1985, 1986; Stoffregen \& Riccio, 1990).

\subsection{Quiet eye vs. technical training}

Supporting task performance by emphasizing perceptual linkages vs. explicit choreography is a clear theme of research on 'quiet eye' (QE). Sports psychologists refer to QE as the stillness of the eyes during aiming tasks, specifically the final gaze fixation to a single location or object in the task for a minimum of $100 \mathrm{~ms}$ within 3 degrees of visual angle immediately before the movement is initiated (Rienhoff et al., 2016). In the case of a golf swing, 'technical training' that communicates an explicit choreography of the arm and leg placement leads to less accuracy than a QE strategy. Expert golfers show more frequent and longer gaze fixations on specific aspects of the putting situation (e.g., golf ball, putting surface, target hole). Novices tend to fixate more introvertedly (e.g., on the clubhead during the backswing phase) and focus on the ball only after it has been hit with the club (Moore et al., 2012; Vickers, 1992, 1996; Vine et al., 2013, 2017). Visual guidance by external focus of attention, that is, focusing on the target leads to a more effective golf swing than technical training of the postures and limb movements composing a golf swing. Indeed, in contrast with technical training, QE training of golf putting leads to improved putting accuracy as well as to a reduction in heart rate and muscle activity at the moment of impact (Moore et al., 2012). Thus, it is believed that instructing athletes by directing their perceptual linkages outward to the visual field supports higher task performance as well as leads to more expert-like movement and physiology.

QE research opens a fascinating vantage point on the perceptual linkages anchoring organisms to their visual contexts and the synergies transforming visual information into action (e.g., KeltyStephen et al., 2020; Mangalam, Lee, et al., 2021; Profeta \& Turvey, 2018). Research on QE has mostly examined the visual-cognitive bases for the relatively more extraverted gaze into the task context (Panchuk \& Vickers, 2006; Rienhoff et al., 2016; Vickers, 2011), and the relationship of QE to the body, the brain, and the nervous system has received relatively less attention (Davids \& Araújo, 2016; Renshaw et al., 2019; Shine et al., 2018). To fill this gap, a 'postural-kinematic hypothesis' has begun to complement the canonical 'visual hypothesis' that $\mathrm{QE}$ was a primarily visual-cognitive mechanism (Gallicchio \& Ring, 2020). So far, this postural-kinematic hypothesis emphasizes only slower movement times, that is, longer duration offering the foundation for longer fixations. This postural-kinematic hypothesis is a constructive first step. In this study, we aim to embed more of the postural dynamics into this hypothesis, beyond simply movement duration. Additionally, instead of reporting on gaze measurements, we will use $\mathrm{QE}$ as a training manipulation and focus on investigating how postural sway supports the effect of the training. 


\subsection{Optic flow and postural sway can support the quiet eye strategy}

A crucial consideration for postural-kinematic contributions to visually guided tasks is optic flow (Stoffregen, 1985, 1986; Stoffregen \& Riccio, 1990). Amidst the 'noisy' fluctuations of the athlete's body, quieting the eyes might leave fewer saccadic interruptions to the coupling between optic flow and postural sway. Optic flow consists of the differential expansion, contraction, or rotation of visible objects and surfaces as an individual moves about. It is specific to both the individual's movement and the contextual layout of objects and surfaces. Indeed, virtual visual displays that impose artificial optic flow also engender anticipatory behaviors (e.g., steering, braking) as if the objects and surfaces entailed by the optic flow were out there-not just for humans but for other animal species and even robots (Serres \& Ruffier, 2017). Although most prominent in locomotion-related contexts, postural sway is no less capable of generating informative optic flow in non-locomotion contexts (Stoffregen et al., 2005). Because research on QE has mostly focused on the fixation of the eye gaze, QE's relationship to optic flow remains a critical knowledge gap (de Oliveira, 2016).

On the face of it, the preceding arguments might ring hollow at first. Certainly, optic flow requires movement, and it could seem wholly unrelated to the issue of visual fixations supporting wholly internal processing of incoming information into a motor program for the body's subsequent control. This gap between QE and optic flow follows closely from ongoing controversy in the literature between ecological vs. information-processing explanations. Optic flow has ecological roots in Gibson's (1979) disavowal of information-processing. Those roots could potentially raise fears that this work might disregard the extensive, diligent work on the information-processing account of QE. Indeed, a rare mention of 'optic flow' in the information-processing approach to QE weds it firmly to 'the teachings of Gibson,' so concerned with invariance as to 'treat every gaze as the same,' and reluctant to respect the fine-grained dynamics of gaze (Vickers, 2009, p. 286). We make full disclosure: the present use of multifractal modeling of postural access to optic flow is inspired by Gibson's appeal to flows and nestings these flows across many scales (Kelty-Stephen \& Wallot, 2017). However, we come to this dialogue with first-hand experience examining the rich heterogeneity of gaze dynamics (e.g., Kelty-Stephen \& Mirman, 2013; Stephen \& Mirman, 2010) and with such interest in cognitive process as for Gibsonians to openly doubt that some of us could be Gibsonians (Raja \& Anderson, 2019). Indeed, our interest in how exploratory behaviors might change perceptual response sets us firmly outside one of the stricter readings of Gibsonian invariance (e.g., Turvey, 1977).

Rather than digging into old trenches, we think that optic flow has a theoretical reach that might pick up where this information-processing account of QE remains incomplete. The theoretical reach of optic flow has grown well beyond Gibson's original intent and usage. For instance, we have already cited cases of optic flow that would have been startling through the strictly Gibsonian lens: information-processing robots use optic flow as well as Gibson's organisms might (Serres \& Ruffier, 2017), and optic flow does not require locomotion but can thrive on postural sway (Stoffregen et al., 2005). In the spirit of making the so far dialogue between ecological and information-processing approaches more constructive, the preceding two examples suggest that optic flow might be much more subtle (e.g., as in standing 'still') but also perfectly amenable to information processing (e.g., as in supporting computational models for controlling action).

Meanwhile, without optic flow to support it, QE's information-processing accounts are incomplete and unmoored from their own explicit requirements and proposals. According to a recent summary, the information-processing view of $\mathrm{QE}$ is that the eye's stillness provides time and attentional resources useful for the preprogramming of movements used in the aimed behavior (Vickers, 2009). This point is defensible but incomplete: visual-cognitive processes benefit only partially from a motor program's stipulation. Even non-ecological explanations have become newly interested in emergent control parameters that sit outside of any simple stipulation by an internal motor 
144

145

146

147

148

149

150

151

152

153

154

155

156

157

158

159

160

161

162

163

164

165

166

167

168

169

170

171

172

173

174

175

176

177

178

179

180

181

182

183

184

185

186

187

188

189

190 program (Vecera et al., 2014). Visual attention is most effective when it is most task- and contextsensitive. The generic usefulness of $\mathrm{QE}$ points to some long-unanswered questions. Are the motor programs implicated in QE flexible and vast enough to accommodate both bodily degrees of freedom and contextual ones? Or are these motor programs elegantly minimal enough to leave room for nonprogrammed factors to support? Neither option is unappealing on its face, but they both reflect an underdeveloped delivery on the information-processing account's proposals about QE. Yes, longer looking times can support stronger, more enriched motor programs (e.g., Witt \& Proffitt, 2008). However, it is not always so simple: either looking time is not always a reliable predictor of motor planning, or the motor program is flexible enough to be rewritten on the fly during the program's execution (Hajnal et al., 2014, 2016).

Specifically, just as it neglects the finer print about its motor programs, QE's informationprocessing account has also neglected its claims about the role of action. The information-processing account offers QE a bidirectional link between gaze, attention, and action (Vickers, 2009). However, whereas this view may respect bidirectionality between attention and gaze (i.e., attentive search for information and looking to what cues attention), the action appears strictly as the outcome passively inheriting whatever the preprogramming entails and only following motor commands. If motor programs generate the consequent swings, then the information-processing approach has yet to elaborate upon two points: first, what bodywide movement coordination supports the postural control allowing the steady visual fixation and, second, how the preprogramming contributes to the externally focused movement. For instance, especially as participants repeat the aiming tasks in front of potentially the same experimental display, the evidence suggests more variety in expert movements than sameness, mainly as they engage visually in the external focus of attention (Morrison et al., 2016). This point raises some questions. If $\mathrm{QE}$ is merely the same sort of fixation every time, irrespective of movement, then why should programs carrying similar, static information give rise to varied bodily execution of the program? If the motor programs that QE supports vary, what is the possible source of variability? In the former case, the programs might underspecify the movements, and in the latter case, the programs would seem to embody more variety than the fixity of the visual scene. The whole issue entailed by the Bernsteinian 'degrees of freedom' concern is that there is no clear programmatic path from intention to bodily execution. Suppose action did participate in the same bidirectional relationship that information-processing approaches to QE observe between attention and gaze. In that case, there might be a way forward, allowing for action to participate in the tuning of programs to ongoing bodily postures and physiological changes throughout repeated behaviors.

To summarize, the gap between QE and optic flow is critical because the informationprocessing approach aligns the fixity of the eyes with the accuracy of aimed behavior. However, there is a little account of the body between the fixations and the accurate behaviors. This omission of the body is immensely curious and potentially problematic because movement exhibits rich variability. It has been difficult for all proposed motor programs to specify accurate goal-directed behavior (Feldman, 2018) and no less difficult when eye movements are implicated (Feldman \& Zhang, 2020). Moreover, if we can leave off old associations and let optic flow continue to take on shapes beyond the original Gibsonian imaginings, we might reinstate the body to an account of QE without any threat of thwarting or disregarding prior art. We aim to rekindle this dialogue between cognitive psychology and ecological psychology, but we aim to do so without requiring disavowal of any mutually opposed pretheoretical commitments.

The present work aims to replicate the beneficial effects of QE on putting performance, and more importantly, to understand how eye height and optic flow might support QE. We used a single session in the style of Moore et al.'s (2012) golf-putting training paradigm to test for rapid attunement to changes in information from optic flow and postural sway. Optic flow centers on an external focus 
of control, aligned with their eye height (e.g., Warren, 1984), and when we perturb eye height, we destabilize optic flow, and the organism needs to learn new relationships between bodily movement and movement of visible surfaces (Frenz et al., 2003). So, we aimed to test whether perturbing the optic -flow parameter of eye height perturbs the effect of QE training in golf putting more so than technical training. In non-golf contexts, for instance, manipulating eye height by adding wooden blocks to participants' shoes alters participants' affordance judgments significantly, leading to an initial underestimation of the optimal climbing height for a stair and sitting height for a chair (Warren, 1984). However, participants adjust to the new eye height and make more accurate decisions shortly afterward (Mark, 1987; Mark et al., 1990). Hence, we expected that adding wooden blocks-(henceforth, 'clogs', to distinguish from 'blocks' of experimental trials) — to the feet might upset putting accuracy in the QE-trained participants initially, but that this accuracy would rebound as participants adjust to new eye height (Rienhoff et al., 2016).

\subsection{Multiscaled organization of postural sway entails a multifractal structure}

Besides manipulating eye height, our expectations about the optic-flow foundation for QE also included a hypothesis about postural sway moderating effects of perturbed eye height. If optic flow could support QE, we expected that QE-trained response to eye height perturbation would depend on postural sway. Indeed, in the affordance research on reattunement to eye-height perturbations, postural sway supported the adaptation of the eye-height relationship to judgments of optimal sitting height (Mark et al., 1990). However, the exact aspects of sway supporting adaptation have been unclear. It was not simply that more sway facilitated this adaptation because, for instance, an 'awkward stance' requiring more compensatory postural adjustments did not support visual adaptation as much as comfortable standing did. An additional challenge in identifying adaptation-supporting aspects of sway could be that sway unfolds across multiple timescales. At longer scales, QE training itself might influence sway by training an explicit use of visual information. At shorter timescales, adding clogs might destabilize posture initially, and then at the shortest timescales, sway might be implicated in adapting to this perturbation of eye height. Hence, judicious accounting of sway's contribution to visual adaptation requires an appreciation of sway's multi-scaled organization.

Then again, the multi-scaled organization of sway may itself reveal a key to predicting-if not explaining - how the movement system adapts its visually-guided actions. Interactions across multiple timescales entail a 'multifractal' structure in sway. Multifractality refers to multiple (i.e., 'multi-') relationships among fluctuations and timescales, with these relationships following power laws with potentially fractional (i.e., '-fractal') exponents. Interactions across timescales engender a nonlinear patterning of fluctuations that multifractal analysis can diagnose and quantify (Ihlen \& Vereijken, 2010). Specifically, multifractal analysis estimates a spectrum of fractal exponents whose width $W_{\mathrm{MF}}$ quantifies "multifractality." When we compare measured series' $W_{\mathrm{MF}}$ to spectrum widths of a set of best-fitting linear models ('linear surrogates') of those series, multifractal evidence of nonlinearity manifests as a significant difference between $W_{\mathrm{MF}}$ for the original series and the average $W_{\mathrm{MF}}$ for linear surrogates. The $t$-statistic, $t_{\mathrm{MF}}$, comparing the original series' $W_{\mathrm{MF}}$ to the surrogates' $W_{\mathrm{MF}}$ serves as an estimate of what we call 'multifractal nonlinearity' (i.e., how much multifractality in the original series is due to nonlinear interactions across timescales).

\subsection{Multifractal nonlinearity predicts perceptual responses to perturbations of task constraints}

Multifractality, $W_{\mathrm{MF}}$, in postural sway is a robust predictor of perceptual responses in both visual and haptic media (Doyon et al., 2019; Hajnal et al., 2018; Teng et al., 2016). Multifractal nonlinearity, $t_{\mathrm{MF}}$, in postural sway predicts perceptual responses as well, not just in the case of nonvisual judgments of length and heaviness of manually-wielded objects (Mangalam \& Kelty-Stephen, 2020) but also in visuomotor tasks (Bell et al., 2019; Booth et al., 2018) and in response to perturbations, such as those 
due to prismatic goggles in a visual aiming task (Carver et al., 2017). Now, we aimed to test whether $t_{\mathrm{MF}}$ could predict the responses to perturbations of eye height in QE-trained golf putting.

We expected that postural sway most adaptive to the perturbation of eye height would show relatively narrow multifractal spectra that would still differ significantly from those of the surrogates. So far, postural sway shows relatively narrower multifractal spectra (i.e., smaller $W_{\mathrm{MF}}$ ) for younger participants (Munafo et al., 2016), those less likely to experience motion sickness (Koslucher et al., 2016), and those focusing on more proximal surfaces in optic flow ( $\mathrm{Li}$ et al., 2020). Hence, greater $W_{\mathrm{MF}}$ in postural sway might counteract effects of QE. All these findings are qualified by a failure of $W_{\mathrm{MF}}$ to correlate with canonical measures of sway like standard deviation. However, postural stability is only ambiguously related to the standard deviation of sway, requiring neither too much nor too little variability in sway (Chen et al., 2008; Rajachandrakumar et al., 2018). Meanwhile, both empirical and theoretical work suggests that $t_{\mathrm{MF}}$ is more closely related to postural stabilizing. Postural stabilization following a perturbation to the base of support shows a rapid reduction of $t_{\mathrm{MF}}$ (Kelty-Stephen, 2018). Theoretical simulation also predicts that random perturbations to individual scales of a nested system lead to narrower multifractal spectra that still differ from linear surrogates' spectra (i.e., smaller $W_{\mathrm{MF}}$ and smaller-yet-significant $t_{\mathrm{MF}}$; Lee \& Kelty-Stephen, 2017). Hence, adapting to the posturally perturbed visual information might involve exhibiting smaller-yet-significant $t_{\text {MF }}$.

\subsection{Golf-putting task and hypothesis}

The participants were instructed to take golf putts towards a circular, hole-sized target painted on a green putting surface in the laboratory-over four blocks of ten trials each. One group received QE training, and the other group received technical training specifying how to move their limbs to make the golf putt. Wooden clogs were attached to the feet of all participants to perturb their eye height. Putting performance was treated continuously in terms of unsigned radial distance from the target (i.e., unsigned radial error).

\subsubsection{Hypothesis-1: Multifractal fluctuations in postural sway will lead an eye height perturbation to interrupt QE-trained performance more so than technical-trained performance}

We hypothesized that the eye-height perturbation due to clogs would lead to lower putting accuracy characterized by higher unsigned radial error following QE training than technical training. We predicted that the clog's effects on QE would depend on the multifractal nonlinearity, $t_{\mathrm{MF}}$, of sway above and beyond any effects of multifractality (i.e., $W_{\mathrm{MF}}$ ) alone (Hypothesis-1a). We also predicted that lower $t_{\mathrm{MF}}$ of sway would be associated with more adaptive responses to the eye-height perturbation, leading to gradual increase and subsequent decay of radial error across trials within the blocks with the clogs on (Hypothesis-1b).

\section{Methods}

\subsection{Participants}

Twenty undergraduate students (11 women, mean \pm 1 s.d. age $=19.5 \pm 1.2$ years, all right-handed, Oldfield, 1971) with normal or corrected vision participated in this study. All participants provided written informed consent approved by the Institutional Review Board (IRB) at Grinnell College (Grinnell, IA).

\subsection{Procedure}

In an hour-long session, the participants completed a single putt on each of 40 trials. Prior to the training trials, half of the participants received QE instructions (Table 1, left column) while the other half received technical putting instruction (Table 1, right column), replicating Moore et al.'s (2012) 
methodology. The technical instructions mirrored the QE instructions but did not describe the eyerelated behaviors, hence mimicking standard golf instruction. After verbally confirming that they understood the instructions, the participants watched a video demonstrating a QE-specific putting stroke (https://www.youtube.com/watch? $\mathrm{v}=\mathrm{lfWuL1klbsY \& feature=youtu.be}$ ) or a standard putting stroke (https://www.youtube.com/watch?v=HOYZn4MYmDI). The researcher directed the QEstrained participants to the key features of the golfer's gaze control and the technically-trained participants to the putting mechanics.

The participant performed the golf putts on a $6 \times 15$ feet standard indoor All Turf Matts ${ }^{\mathrm{TM}}$ artificial turf mat using a 1.68-inch diameter PING Sigma 2 Anser Stealth ${ }^{\mathrm{TM}}$ putter and regular-sized white golf balls. The participants completed straight putts ten feet away towards a 2.5-inch diameter circular orange dot, designed to fit the golf ball. This format allowed for more stringent measurement and gave the participants a more focused target.

The participants completed 20 training trials (treated as two blocks of 10 trials per block in regression modeling). Following a short 5-minute break, the participants completed two blocks of ten test trials per block (i.e., 20 test trials total) in the absence of instructions. The participants were randomly assigned to complete either their first or second block of ten trials while wearing 5-cm-raised wooden blocks (i.e., clogs) attached to their shoes (they completed the other block of ten test trials under the same conditions as the 20 training trials). Before the testing began, the participants were allowed to walk around and adjust to the clogs to reduce fall risk.

\subsection{Measures}

The task performance was defined in terms of the radial error (the distance from the target at which the ball stopped, in feet) and the number of putts successfully 'holed.' Because the target was not a real hole, the putt was counted as holed in either of two events: the ball came to rest within the orange dot on the floor or within 2 feet directly beyond the hole (Cooke et al., 2010; Vine et al., 2011). Postural sway was measured using a smartphone with the Physics Toolbox Suite application (http://vieryasoftware.net) strapped to the participants' lower back. The app recorded the acceleration of the torso along $x$-, $y$-, and $z$-dimensions at $100 \mathrm{~Hz}$. An experimenter manually started and stopped recording for each putt. Recording began after the participant set up over the ball and stopped once the ball stopped moving.

\subsection{Multifractal analysis}

\subsubsection{Multifractal spectrum width of accelerometer displacement series}

For each putt, we computed an accelerometer displacement series for multifractal analysis as follows. The accelerometer data encodes an $N$-length series of accelerations of the torso along $x$-, $y$-, and $z$ dimensions centered on an origin on the smartphone. A displacement series of length $N-1$ was computed by: 1) taking the first differences for the variable (e.g., if we use the letters $i$ and $j$ to indicate individual values in sequence, $x_{\text {diff }}(j)=x(i)-x(i-1)$ for each $i$ th value of $x$, for $2<i<N$, $1<j=i-1<N-1,2)$ squaring and summing the differences of each dimension's displacements (i.e., $x_{\text {diff }}^{2}+y_{\text {diff }}^{2}+z_{\text {diff }}^{2}$ ), and 3) taking the square root of this sum (i.e., $\sqrt{\left(x_{\text {diff }}^{2}+y_{\text {diff }}^{2}+z_{\text {diff }}^{2}\right) \text { ). }}$.

Multifractal spectra $f(\alpha)$ were estimated for each accelerometer displacement series, using Chhabra and Jensen's (Chhabra \& Jensen, 1989) method. The first step was to partition the series into subsets (or 'bins') of various sizes, from four points to a fourth of series' length (Fig. 1, left). The second step was to examine how the proportion of displacements within each bin changed with bin size or timescale (Fig. 1, right). The third step was to estimate two distinct exponents: an exponent $\alpha$ describing how the average proportion grows with bin size and an exponent $f$ describing how 
Shannon's (Shannon, 1948) entropy of bin proportion changes with bin size. The later steps were all iterations of this third step, calculating a mass $\mu$ by applying an exponent $q$ to bin proportions and using $\mu(q)$ to weight proportions selectively according to proportion size. $q$ greater than 1 weights larger-proportion bins, and $q$ lesser than 1 weights smaller-proportion bins (Fig. 2, left). $q$-based mass $\mu(q)$-weighting generalizes single $\alpha$ and $f$ estimates from earlier steps into continual $\alpha(q)$ and $f(q)$ with $\alpha(q)$ describing how mass-weighted average bin proportion changes with bin size and $f(q)$ describing how Shannon's entropy of bin masses $\mu(q)$ with bin size (Fig. 2, top right).

Multifractal analysis quantifies heterogeneity as variety in $\alpha(q)$ and $f(q)$. The ordered pairs $(\alpha(q), f(q))$ constitutes the multifractal spectrum (Fig. 2, bottom right). We included $\alpha(q)$ and $f(q)$ only when mass-weighted proportions were defined and when both of their corresponding relationships to bin size correlated at $r>0.995$ on logarithmic plots, for bin sizes $4,8,12, \ldots N / 4$ and $-300<q<$ 300 , where $N$ is the number of samples in the series.

\subsubsection{Surrogate comparison}

Multifractal spectrum width is sensitive to nonlinear interactions across timescales as well as to linear temporal structure. Hence, evidence of nonlinear interactions across timescales requires comparing the original series' multifractal spectrum width $W_{\mathrm{MF}}$ to those computed for a sample of linear surrogate series (Fig. 3). These linear surrogate series retain the same value as the original series but in a different order that preserves the original series' linear autocorrelation but destroys original sequence (i.e., iterative amplitude-adjusted Fourier-transform or IAAFT (Ihlen \& Vereijken, 2010; Schreiber \& Schmitz, 1996); Figs. $4 a$ to $d$ ). Comparing $W_{\mathrm{MF}}$ of the original and surrogate series (Figs. $4 e, f$ ) produces a $t$-statistic $t_{\mathrm{MF}}$, a measure of nonlinear interactions across timescales that is standardized across series with differing linear temporal structure. $t_{\mathrm{MF}}$ was computed for each original series.

\subsection{Mixed-effects modeling}

The data were submitted to two kinds of mixed-effect modeling, namely, logistic and Poisson, using the glmer() function in $R$-package lme4 (Bates et al., 2007). The subsequent subsections provide details of the predictors and the models.

\subsubsection{Effects of time}

The effects of time were modeled using trial number within a block (trial ${ }_{\text {block}}$ ), block number (block), and interactions of trial number with block number (trial ${ }_{\text {block }} \times$ block), and trial number across blocks $\left(\right.$ trial $\left._{\text {exp }}\right)$. The model used orthogonal polynomials of each of both predictors up to $3^{\text {rd }}$ order (i.e., cubic). The primary reason for the polynomial terms was to reveal how and when the perturbation of wearing clogs might manifest and consequently subside. Cubic polynomials allow the simplest way to accomplish this goal by allowing a peak and subsequent decay to a flat, quiescent portion as part of the same continuous function. Additionally, unlike the quadratic function, the cubic peak is not required to be symmetric and centered on the midpoint. Hence, the cubic function controls for any nonlinearities in the normal course of trials without such perturbations. Whereas block is a linear effect across blocks, including trial $l_{\exp }$ in the model allowed controlling for nonlinear change across blocks.

There are two important features to note about the treatment of time in this model. First, the Poisson model encoded both classifications of trial numbers (i.e., trial $l_{\text {exp }}$ and trial $_{\text {block }}$ ) in terms of an orthogonal $3^{\text {rd }}$-order polynomial, using the function 'poly' in $R$, to include the linear, quadratic and cubic growth of trial number with all three terms phase-shifted so as to be uncorrelated with one another. Second, the lack of model convergence limited the appearance of block, such that block was neither treated nonlinearly nor in interaction with perturbation. Attempting to include the orthogonal $3^{\text {rd }}$-order polynomials for block number led to a failure of model convergence, so block only appeared 
369

370

\section{1}

as a linear term. The model also failed to converge when it included interactions of block and perturbation because these two terms were strongly collinear.

\subsubsection{Effects of manipulations}

Manipulations included quiet-eye instruction, $\mathrm{QE}(\mathrm{QE}=1$ for receiving $\mathrm{QE}$ instruction, and $\mathrm{QE}=0$ for not receiving $\mathrm{QE}$ instruction) and perturbation of eye height, perturbation (perturbation $=1$ for all ten trials during the block when participants wore the clogs, and perturbation $=0$ for all other trials).

\subsubsection{Sway: Linear predictors}

The accelerometer data encodes accelerations of the torso along $x$-, $y$-, and $z$-dimensions. Any single posture corresponds to a specific point in this 3D space. Hence, sway corresponds to the deviation of this 3D acceleration around the mean posture. The present analyses encoded this variability as $M S D_{\text {acc }}$ or $R M S_{\text {acc, }}$ depending on which predictor supported a convergent model, along with mean and standard deviation of 3D displacement (Mean $n_{\text {disp }}$ and $S D_{\text {disp }}$, respectively).

\subsubsection{Regression modeling}

A mixed-effects Poisson regression modeled the radial error (in inches) of all misses, with makes as defined above treated as having zero radial error. Predictors included QE $\times$ perturbation $\times$ trial $_{\text {block }} \times$ $t_{\mathrm{MF}}$ to our hypothesis by specifically addressing trials within each block, QE $\times$ trial block $_{\text {and }} \mathrm{QE} \times$ trial $_{\text {exp }}$ to control for differences in learning due to QE across a linear progression of block; a nonlinear function of trials, $\mathrm{QE} \times$ perturbation $\times W_{\mathrm{MF}} \times t_{\mathrm{MF}}$, to control for the effects of multifractal spectral width and its relationship with $t_{\mathrm{MF}}$; and $M S D_{\mathrm{acc}} \times M e a n_{\mathrm{disp}} \times S D_{\mathrm{disp}}$ to control for the effects of linear description of sway. Modeling included lower-order interactions and component main effects (e.g., Allison, 1977).

\section{Results}

\subsubsection{Non-multifractal effects on radial error}

This section details the non-multifractal effects described in Supplementary Table S1 and effects preliminary to the test of our hypothesis in Table 2. This model of radial error found a significant main effect of QE training, specifically, a negative effect $\left(b=-4.40 \times 100\right.$, s.e.m. $\left.=4.81 \times 10^{-1}, p<0.0001\right)$ indicating that QE did promote greater accuracy in terms of proximity of the ball's final position to the hole. Radial error grew linearly and quadratically across trials but showed negative cubic variation with trial-both for trials across the experiment and within a block (Fig. 5a). The QE strategy prompted steady decay of error across the experiment, canceling out the linear and cubic pattern of error over trials within a block (Fig. 5b). The perturbation of eye height elicited no main effect but increased the growth of error over trials within a block, with the QE instruction accentuating this nonlinear growth of error later in the block. The higher-order interaction $M S D_{\text {acc }} \times M e a n_{\text {disp }} \times S D_{\text {disp }}$ and its main component effects and lower-order interactions showed mostly negative effects (only two of the twoway interactions showed positive coefficients).

\subsubsection{Total multifractality of sway, $W_{M F}$, increased radial error, but multifractality due to} nonlinearity, $t_{\mathrm{MF}}$, attenuated radial error at the beginning and the end of blocks

No matter what portion of multifractality is attributable to linear or nonlinear structure, the total width $W_{\mathrm{MF}}$ of original series' multifractal spectra is associated with greater radial error $\left(b=1.80 \times 10^{0}\right.$, s.e.m. $\left.=1.29 \times 10^{-1}, p<0.0001\right)$. We did not find any main effect of multifractality due to nonlinearity, $t_{\mathrm{MF}}$, nor any interaction of $W_{\mathrm{MF}} \times t_{\mathrm{MF}}$. However, the negative effect for $t_{\mathrm{MF}} \times \operatorname{trial}_{\text {block }}$ (Quadratic; $b=-$ $8.93 \times 10^{-2}$, s.e.m. $=1.15 \times 10^{-2}, p<0.0001$ ) indicated an association between multifractality due to 
412

nonlinearity with a significant reduction of the positive quadratic profile for radial error over trials

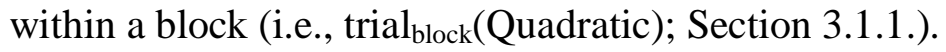

\subsubsection{Testing Hypothesis-1a: Perturbation of eye height increased radial error only in combination with QE training, with greater multifractality due to nonlinearity $t_{\mathrm{MF}}$, or with both}

The multifractality of sway promoted the tendency of QE to increase perturbation-related radial error. Perturbation showed a significant effect through the interaction of perturbation $\times W_{\mathrm{MF}}\left(b=-1.50 \times 10^{0}\right.$, s.e.m. $=3.12 \times 10^{-1}, p<0.0001$ ) canceled out the positive effect of $W_{\mathrm{MF}}$ on error (Section 3.1.2), suggesting that the perturbation reduced the error-eliciting effect of $W_{\mathrm{MF}}$. The interaction $\mathrm{QE} \times W_{\mathrm{MF}}$ reduced error due to multifractality by roughly half $\left(b=-1.04 \times 10^{0}\right.$, s.e.m. $\left.=2.03 \times 10^{-1}, p<0.0001\right)$, suggesting that QE promoted greater accuracy but only in the absence of perturbation. However, the higher-order interaction with multifractality indicates that QE increased the error attributable to the perturbation. For instance, the $\mathrm{QE} \times$ perturbation $\times W_{\mathrm{MF}}\left(b=2.12 \times 10^{0}\right.$, s.e.m. $\left.=4.91 \times 10^{-1}, p<0.0001\right)$ reverses the apparently beneficial error-reducing interaction effect of perturbation $\times W_{\mathrm{MF}}$. Hence, QE reinstated the error due to total multifractality (Section 3.1.2), and it did so only in the face of the perturbation.

Above and beyond the effects of $W_{\mathrm{MF}}$ in moderating the interaction effect of $\mathrm{QE} \times$ perturbation, $t_{\mathrm{MF}}$ promoted the error-increasing effect of perturbation. Specifically, besides the moderating effect of $\mathrm{QE}$ on the effect of perturbation $\times W_{\mathrm{MF}}$, perturbation elicited even greater radial error with increases in $t_{\mathrm{MF}}$ (perturbation $\times W_{\mathrm{MF}} \times t_{\mathrm{MF}}: b=1.54 \times 10^{-1}$, s.e.m. $=3.15 \times 10^{-2}, p<0.0001$ ). QE did reverse this effect $\left(\mathrm{QE} \times\right.$ perturbation $\times W_{\mathrm{MF}} \times t_{\mathrm{MF}}: b=-2.21 \times 10^{-1}$, s.e.m. $\left.=4.97 \times 10^{-2}, p<0.0001\right)$. However, $\mathrm{QE}$ also increased error in combination with perturbation and $t_{\mathrm{MF}}$ even in the absence of any moderating effect of $W_{\mathrm{MF}}\left(\mathrm{QE} \times\right.$ perturbation $\times t_{\mathrm{MF}}: b=8.62 \times 10^{-3}$, s.e.m. $\left.=4.18 \times 10^{-3}, p<0.05\right)$. This result supports Hypothesis-1a.

Despite the absence of a simple $\mathrm{QE} \times$ perturbation interaction, the eye height perturbation produced a greater model-predicted increase in radial error for QE-trained participants. To illustrate this point, we can compare the model predictions for participants in each condition with high and low $W_{\mathrm{MF}}$ and $t_{\mathrm{MF}}$ (with 'low' and 'high' values defined as $1^{\text {st }}$ and $3^{\text {rd }}$ quartiles). For participants with 'low' $W_{\mathrm{MF}}$, the eye height manipulation prompted $2.27 \%$ to $11.14 \%$ increase in radial error in QE-trained compared to technical-trained participants for low to high $t_{\mathrm{MF}}$, respectively. For participants with 'high' $W_{\mathrm{MF}}$, the eye height manipulation prompted $8.80 \%$ to $18.23 \%$ increase in radial error in QE-trained compared to technical-trained participants for low to high $t_{\mathrm{MF}}$, respectively.

\subsubsection{Testing Hypothesis-1b: the QE group with greater multifractal nonlinearity in sway showed a greater initial but progressively slower increase in radial error due to the perturbation of eye height}

The interactions of $\mathrm{QE} \times$ trial $_{\text {block }}$ with multifractal nonlinearity followed a similar but weaker pattern as the interactions of $\mathrm{QE} \times$ trial $_{\text {block }}$ with perturbation. Much like the perturbation of eye height, the interaction of QE with $t_{\mathrm{MF}}$ accentuated the radial error late in a block of ten trials $\left(\mathrm{QE} \times\right.$ trial $\left._{\text {block }} \times t_{\mathrm{MF}}\right)$ : it weakened the linear term $\left(b=-3.73 \times 10^{-2}\right.$, s.e.m. $\left.=1.46 \times 10^{-2}, p<0.05\right)$, contributing to a negativequadratic peak in the middle of the block $\left(b=-1.85 \times 10^{-1}\right.$, s.e.m. $\left.=1.66 \times 10^{-2}, p<0.0001\right)$ and a positive cubic increase at the end of the block $\left(b=1.13 \times 10^{-1}\right.$, s.e.m. $\left.=1.73 \times 10^{-2}, p<0.0001\right)$. Hence, $t_{\mathrm{MF}}$ and perturbation showed independent effects on the progression of error across trials in block.

However, the similarity of these effects independently on error across trials within a block did not extend to their interaction. Indeed, most relevant to testing Hypothesis- $1 \mathrm{~b}$ is that the interaction of perturbation and $t_{\mathrm{MF}}$ served to speed up the growth of error, and QE slowed it down across the block. Coefficients for perturbation $\times$ trial $_{\text {block }} \times t_{\mathrm{MF}}$ indicated that in the face of perturbation, multifractal nonlinearity removed the observed peak in error due to earlier negative quadratic effects $\left(b=3.83 \times 10^{-}\right.$ 
${ }^{1}$, s.e.m. $\left.=5.28 \times 10^{-2}, p<0.0001\right)$ and increased error across the linear $\left(b=4.07 \times 10^{-1}\right.$, s.e.m. $=$ $\left.5.20 \times 10^{-2}, p<0.0001\right)$ and cubic terms $\left(b=5.39 \times 10^{-1}\right.$, s.e.m. $\left.=4.45 \times 10^{-2}, p<0.0001\right)$. On the other hand, coefficients for perturbation $\times$ trial $_{\text {block }} \times t_{\mathrm{MF}}$ indicated that, in the face of perturbation for the QE participants, the linear increase was shallower $\left(b=-2.71 \times 10^{-1}\right.$, s.e.m. $\left.=6.11 \times 10^{-2}, p<0.0001\right)$, the positive quadratic growth further removed the observed peak in error $\left(b=4.24 \times 10^{-1}\right.$, s.e.m. $=6.14 \times 10^{-}$ $\left.{ }^{2}, p<0.0001\right)$, and the cubic term was negative $\left(b=-7.90 \times 10^{-1}\right.$, s.e.m. $\left.=5.79 \times 10^{-2}, p<0.0001\right)$.

The outcome of these differences distinguishes how multifractal nonlinearity predicted changes in adaptive responses to the perturbation of eye height. Greater $t_{\mathrm{MF}}$ exhibited less adaptive response to the perturbation in the QE case (i.e., initially greater error and a smaller change in error across trials), and less-but-still-nonzero $t_{\mathrm{MF}}$ predicted a more gradual appearance of error and then a subsequent decay of this error (Fig. 5b), suggesting better adaptation. It is evident in figure $5 b$ that although the error in the high- $t_{\mathrm{MF}}$ cases with the perturbation was smaller than the delayed increase in the low- $t_{\mathrm{MF}}$ cases with the perturbation, the high- $t_{\mathrm{MF}}$ cases showed more sustained error and no adaptation to the perturbation relative to high $-t_{\mathrm{MF}}$ cases without the perturbation. This result supports Hypothesis- $1 \mathrm{~b}$.

\section{We include example plots of model predictions for individual participants (Fig. 6).}

\subsection{Logistic regression of 'makes' vs. misses showed less sensitivity to effects}

Poisson regression may be too ornate for some readers. However, in contrast to Poisson modeling of a continuous outcome measure of performance, the investment of logistic regression to model dichotomous variable limits the number of valid predictors (e.g., van Smeden et al., 2016) and so affords us less statistical power with which to test for changes in the performance. A mixed-effect logistic regression model of successful putting (i.e., the 'makes') returned the coefficients reported in Table 3. Predictors for $t_{\mathrm{MF}}$ and for interactions of $\mathrm{QE}$ with all terms other than perturbation failed to improve model fit significantly and so were omitted from the final model. This model failed to return an effect of QE $(b=0.20$, s.e.m. $=0.30, p=0.51)$, of the perturbation alone $(b=-0.22$, s.e.m. $=0.43, p$ $=0.60)$ or the interaction of $\mathrm{QE} \times$ perturbation $(b=-1.16$, s.e.m. $=0.65, p=0.07)$.

We found effects of experience and trial-by-trial sway. Block showed a positive effect $(b=$ 0.24 , s.e.m. $=0.13, p=0.06$ ), suggesting that odds of a successful putt was 1.27 times higher with each successive block of trials. $R M S_{\text {acc }}$ showed a positive effect $(b=4.05$, s.e.m. $=1.34, p<0.01)$, and total multifractality $W_{\mathrm{MF}}$ showed a negative effect $(b=-7.25$, s.e.m. $=2.94, p<0.05)$, suggesting that successful putts were more likely with more variable and less multifractal accelerations of sway. These effects resembled some of those found in the Poisson regression model, but it appears that the continuous measure of radial error afforded more sensitivity within which to model significant fixed effects.

\section{Discussion}

We tested the effects of postural stability and its endogenous multifractal fluctuations on a continuous outcome of putting performance (radial error) with and without QE training. We hypothesized that QEtrained participants' response to an eye height perturbation would depend on the multifractality attributable to nonlinear interactions across scales, $t_{\mathrm{MF}}$. That is, more multifractal fluctuations in postural sway would make eye height perturbation a greater threat to QE-trained performance but not to technically trained performance. Specifically, we predicted that lower estimates of $t_{\mathrm{MF}}$ would coincide with a more adaptive response, i.e., with slower growth of radial error and subsequent decay of radial error over trials. Results supported this hypothesis: when we perturbed eye height, accuracy with QE was associated with reducing multifractal nonlinearity in postural sway. This model included a negative main effect of QE training, indicating more accurate putts in radial error. However, despite 
503

504

505

506

507

508

509

510

511

512

513

514

515

516

517

518

519

520

521

522

523

524

525

526

527

528

529

530

531

532

533

534

535

536

537

538

539

540

541

542

543

544

545

546

547

548

549 the absence of a simple interaction of QE with clogs, QE's contingency on multifractal aspects of postural sway entailed a greater decrement in accuracy for QE-trained participants following eye height perturbation.

A limitation of these results is that our focus on the postural support of QE-trained performance came at the expense of focusing on gaze tracking. We used QE training as a manipulation, but we failed to include a manipulation check. The absence here of gaze-tracking data leaves us unable to explain with complete confidence why a logistic model of putting performance construed dichotomously (e.g., as 'makes' vs. 'misses') failed to find a significant effect of QE. On the one hand, some participants may have followed $\mathrm{QE}$ instruction incompletely. On the other hand, a good reason why the main effect may not appear is the brevity of our design: whereas recent analogues of our research design already in the literature required participants to conduct 4000 putts over 7 days (Moore et al., 2012), we only sampled 40 putts in one hour-long session. Radial error may be sufficiently sensitive to show QE's reliable effect in a more compact design than dichotomous measures. Future research would no doubt benefit from incorporating gaze-tracking into similarly compact experimental designs.

These results offer two major insights for research into perception-action in visual aiming tasks. First, it shows that QE training has roots in postural sway, vindicating and elaborating earlier proposals that quiet eye is not simply about stabilizing the eyes (Liphart et al., 2015; Williams, 2016). Indeed, it has long been known that simply stabilizing the retinal position compromises the persistence of a visual image (Barlow, 1963; Coppola \& Purves, 1996; Ditchburn \& Ginsborg, 1952). And contrary to a colloquial understanding of 'quiet eye,' more recent work confirms that those small movements within gaze fixations called 'microsaccades' might prevent fading (Martinez-Conde et al., 2006) and support visual attentional processes (Krueger et al., 2019). We might draw a comparison between the 'quiet' suggested by 'quiet eye' and that indicated by 'quiet standing.' To be precise, we mean to draw the comparison of 'quiet eye' specifically only to 'quiet standing' in the case of instructing participants to 'stand as still as possible' (i.e., rather than to 'stand as relaxed as possible'; (Houdijk et al., 2015). Both 'quietudes' provide the needed instruction to a participant or athlete to 'please move as little as possible,' but below the polite clarity for verbal instruction, both 'quietudes' depend upon a rich texture of fluctuations. Throughout the body, fluctuations support exploration during the 'still as possible' quiet standing (I.-C. Lee et al., 2019; Murnaghan et al., 2016; Palatinus et al., 2013, 2014).

Here, the theoretical ground may shift underfoot as we try to see a path through these considerations. Do we mean to say that the achievement of a precision demand could be exploratory? At first blush, it could sound absurd that something not moving is also exploring. However, here, we may hit upon a deeper divergence between ecological vs. information-processing perspectives on QE. To the point, it is true that fixing the gaze and standing as still as possible are themselves accomplishments of motor control. However, this achievement does not entail that either quiet eye or quiet stance are the results. Unless we are much mistaken, it is the external focus and the intention of getting the ball into the net or hole or target that warrants quiet eye in the first place. That is to say, the quiet eye is a means to an end, and the end is never just quiet eye.

Precision might invite the appearance of achieving fixity, but achieving fixity might be no guarantee against exploration. For a contrary example, 'static holding' is a haptic case of holding an object 'as still as possible,' and it is widely recognized as a valid exploratory procedure for haptic perception (Burton \& Turvey, 1990; Carello et al., 1992; Lederman et al., 1996). The only debates are what kind of information this apparent-fixity mode of exploration has access to. Crucially, apparent fixity is not necessarily actual fixity, and while there is no need for the body to explore the quiet-eye achievement of ocular fixity, it would strike us as a strange eventuality to rule quiet-eye strategy out as a strategy pertaining to exploration. Quiet eye resembles to us an ocular version of static holding that 
550

551

552

553

554

555

556

557

558

559

560

561

562

563

564

565

566

567

568

569

570

571

572

573

574

575

576

577

578

579

580

581

582

583

584

585

586

587

588

589

590

591

592

593

594

595

596

holds the visual image as close as possible. As noted above, despite their apparent fixity, fixations are a class of eye movements, and these fixational eye movements are exactly what the quiet-eye strategy uses to build a motor program. Certainly, if the QE strategy is collecting information that supports the construction of a motor program, ruling quiet eye out as a form of exploration would strike us as a problematic move. For instance, consider the pioneering astronomers gazing up at the heavens, aiming a telescope as quietly as possible at a star or planet. Can we tell them that, no, because they are engaged in precision work, they are not exploring? We suspect not. Again, to summarize this point: a precision demand can change motor behavior (Balasubramaniam et al., 2000), but that change is not itself the end of the story. Despite experimenter intentions to train it, quiet eye is not a goal in itself but only a strategy for informing the participant's accurate behavior beyond their bodies for precision wherever they direct their external focus.

Of course, the preceding proposal that QE is exploratory remains conjecture that reaches beyond the current evidence. Demonstrating an exploratory structure in QE will need empirical evidence from future research to back up the preceding metaphor and analogy. Specifically, empirical validation would require at least one of the following: (1) eye movement data indicating exploratory movements within the fixation time that are similar or relational to those from the haptic or postural research, (2) performance outcome data that indicates information is obtained that is of value during the quiet eye fixation period, and/or (3) self-reporting of the subjects (perhaps quantified through perceptual judgments) indicating information gained following QE training that differs from those who do not go through the training. We look forward to future research to the opportunity to test these ideas and revisit the question of whether QE is exploratory behavior.

We may summarize this first insight as follows: The value of QE is not necessarily a net reduction of fluctuations but rather a way to train athletes to orient their movements towards the optic flow generated by postural sway. Whereas the postural-kinematic hypothesis had previously only referred to longer movement duration (Gallicchio \& Ring, 2020), we now report that movements are not merely slower but evince a sort of nonlinearity constricting the degrees of freedom across scales of the movement-system hierarchy. In one of the subsections below, we discuss how this orienting towards optic flow might coordinate with mechanisms invoked by the more elaborate visual hypothesis (e.g., Gallicchio et al., 2016, 2017).

The second insight from the present results is that the multifractality in sway is an essential aspect of postural adaptations contributing to visually-guided action. It was already known that damping out postural perturbations could occasion a ratcheting down of multifractality in postural sway (Kelty-Stephen, 2018). The present results indicate that this reduction of multifractality is associated with more accurate responses in visually-guided aiming. Note that this reduced multifractality remains different from corresponding surrogates. Hence, nonlinear interactions across scales can constrict movement variability, making reduced multifractality more adaptive. This distinction is a departure from any shorthand presumption that 'more multifractal is better' supported by early studies of heart rate variability (HRV; Ivanov et al., 2001). Indeed, this departure reflects a growing clarity about the value of multifractal fluctuations for predicting and explaining outcomes in perception-action.

\subsection{Clarifying the value of multifractal fluctuations for perceiving-acting systems}

The shorthand 'more multifractal is better' presumptions from early studies on HRV (Ivanov et al., 2001) have always underestimated the more elaborate systematicity in the physiology of the human movement system with upright posture. Early work proposed that a 'loss of [fractal or multifractal] complexity' would coincide with aging or with pathology (Lipsitz \& Goldberger, 1992). Indeed, early evidence showed that less healthy outcomes co-occurred with more-than-fractal levels of temporal correlation (Lipsitz, 2002). However, the task has always been known to influence multifractality in 
597 upright posture: multifractality of quiet standing can reduce with pathology (Morales \& Kolaczyk, 2002; Shimizu et al., 2002), but multifractality in gait can also increase with pathology (MuñozDiosdado, 2005), as well as with walking speeds faster or slower than self-selected comfortable speed (West \& Scafetta, 2003). Over the lifespan, the maturation of gait involves loss of multifractality, beginning with the stabilization of toddler gait into young adult gait and continuing into older age (Ashkenazy et al., 2002). And this lifelong progression of multifractality in gait actually is at odds with more recent evidence that multifractality in HRV remains stable with healthy aging (Schmitt \& Ivanov, 2007).

Militating strongly against simple 'more multifractal is better' kind of presumptions are a variety of distinctions. Some of these distinctions may sound patently obvious but need recognition if we want to identify for what, after all, multifractality is good. These distinctions include, for instance, that hearts are not upright bipedal bodies, that age is not itself a pathology, and that walking is not standing. The remaining distinctions are more subtle. In the promise of fractal/multifractal analysis, the discourse about 'complexity,' 'dynamical stability,' and the role of fractality/multifractality in 'optimality' (Friston et al., 2012; Oftadeh et al., 2014; Schlesinger \& Klafter, 1986) has made no claims on all definitions of optimality and so has not been responsible for acknowledging that some multifractality is not optimal (Reynolds, 2015). Indeed, studies in behavioral sciences have always justified the capacity of fractal variety to offer new insights (Diniz et al., 2011; Dixon et al., 2012). Any notion of a privileged level or direction of fractal variety (Kello, 2018) might be best understood as a reaction to critical, unreceptive perspectives resistant to seeing any fractality or multifractality appear in behavioral-science discourse at all (Wagenmakers et al., 2012). Measurements may wax and wane in their multifractal structure. The only case in which we think more multifractality is always better than less is in its inclusion as a construct in the scientific discourse on the coordination of perceiving-acting systems.

\subsection{The value of multifractal fluctuation is specific to task constraints}

What is coming into focus is that the value of nonlinearity-driven multifractality depends on task context. More multifractal nonlinearity is adaptive when tasks invite exploration and anticipation. Less multifractal nonlinearity is adaptive when the task involves constraining behavior, drawing degrees of freedom inward around a base of support. As an example of the former, accumulating information as a reader can require keeping an open mind to follow the discourse wherever it may lead, and more multifractality $t_{\mathrm{MF}}$ in the pacing of word-by-word reading supports more fluent reading if the story has a twist in its plot (Booth et al., 2018). Similarly, more multifractality $W_{\mathrm{MF}}$ in circle-tracing behaviors make for poor precision of tracing but is associated with better performance on neurophysiological tests of flexibility with rule switching (Kelty-Stephen et al., 2016). The freewheeling benefits of more multifractality can also compete alongside the constraint-, repetition-promoting benefit of less multifractality. In a Fitts task, greater multifractality $t_{\mathrm{MF}}$ in hand and head movements predicted less stable contact with the targets, but it became a predictor of more stable contact when participants had the diffuse warning that they might be asked to close their eyes and continue the task (Bell et al., 2019). Hence, what would have upset task performance became a resource for exploring the task space for the eventuality of a major loss of sensory information. And now, we see that a less multifractal postural system can be more effective (e.g., smaller $t_{\mathrm{MF}}$ in Kelty-Stephen (2018); and smaller $W_{\mathrm{MF}}$ in Koslucher et al. (2016) and Li et al. (2020)) and, as present findings indicate, more accurate in how it orients behaviors to a visually-guided aiming task.

Some of the task-specificity remains unclear, e.g., manual wielding of an object is more accurate with greater multifractality in postural sway, but hefting an object to perceive heaviness compared to a reference object is more accurate with less multifractality (Mangalam \& Kelty-Stephen, 2020). This difference could have to do with the qualities of length and heaviness, but the simpler 
644

\section{5}

646

interpretation could be that intending to perceive length directs attention away from the center of pressure and intending to perceive heaviness directs attention inwards toward the center of pressure, lending length perceptions and heaviness perception to less and more postural constraint, respectively. This issue of directing attention is again a reason we will need to consider how to link this posturalkinematic work on QE back with the visual-hypothesis work on QE.

The fact that reduced multifractality can be beneficial offers a unique distinction between possibly two related but distinct interpretations of multifractal results: one as a biomarker of pathology and the other as an adaptation. Indeed, the loss of multifractality can be a biomarker for diagnosing a pathology (Meyer et al., 2003). The physiological wisdom informing this usage is that the heart has to be, in effect, poised to absorb and rebound from perturbations, avoiding fragility due to insult at any single characteristic scale (e.g., Lipsitz \& Goldberger, 1992). Implicit in this wisdom is that, within the healthy body, the heart's task is deeply anticipatory and exploratory, much like those task settings in which whole organisms benefit from more multifractality. However, concluding that loss of multifractality is strictly symptomatic of postural systems with deficits (Shimizu et al., 2002) may be premature. What looks like a deficit may sometimes be a creative constraint, built and used to solve a task problem (Harrison \& Stergiou, 2015; Holt et al., 2010; Vaz et al., 2017). The present results indicate that reduced multifractality could be an adaptive response preserving stability and promoting task performance in the face of abrupt change in the organism's mechanical relationship with the base of support. Hence, far from being the signature of diseased posture, reductions in multifractality that maintain a significant $t_{\mathrm{MF}}$ may be an advantageous loss of multifractality. Though more commonly known for their capacity to amplify variability, nonlinear interactions across scales acting contextsensitively may also restrain motoric degrees of freedom so as to achieve the task goal.

Different task constraints prompt different modes of movement variability, and if multifractal depictions of the body seem to physicalize or mechanize the movement system, they do so only in the way that nonlinear-dynamical physical mechanisms might better explain context-sensitive behavior. Surely, good-faith pursuit into nonlinear-dynamical complexity should avoid any catch-all simplification (e.g., 'more is better, less is worse') and keep a critical eye on the reference frames of nonlinear-dynamical results. Nonlinear dynamics has long profited from considering its measures in different reference frames: experimentally breaking apart the coincidence of relative-phase in spatial frames from relative-phase in muscular frames revealed novel, more generic structure in the HakenKelso-Bunz (1985) law for multi-limb coordination (Park \& Turvey, 2008). Acknolwedging the taskbased reference frames for our interpretation of multifractal estimates may be equally crucial for generalizing predictions for a multifractal foundation for perception-action. Low multifractality in the heart signals a bad prognosis to the clinician when the task is cardiovascular circulation, but low multifractality in posture supports good task performance in visually-guided aiming, particularly for an organism beset by malevolent experimenters uprooting their optic flow.

\subsection{The value of multifractality is also specific to different tissues of the body.}

Just as different tissues of the same body can differently support the same task, we expect that the multifractal structure spanning these different tissues supports overarching integrity to the organismallevel behavior differently (Stoffregen et al., 2017). Here, comparing present results with similar work using a relatively less-mechanical, visual perturbation (Carver et al., 2017) may tee up considerations for future work that could elaborate an explanation of QE by integrating the postural-kinematic hypothesis with the visual hypothesis. The present results resemble those of Carver et al. (2017) who found that, without any training, more multifractality in head sway led to greater error in a lowdifficulty Fitts task (compare $W_{\mathrm{MF}}$ in Table 2) but led to smaller error in the case of a visual perturbation (compare perturbation $\times W_{\mathrm{MF}}$ in Table 2). So, multifractality in head sway became a stabilizing, accuracy-promoting asset to task performance when the target was far out of reach. Future 
691

692 research could probe the visual hypothesis for QE for multifractal foundations, and based on Carver et al.'s (2017) results, we predict that QE-trained performance would improve with greater multifractality in head sway or eye movements. Monofractal analysis of eye movements has successfully predicted visual attention to text (Wallot et al., 2015), and multifractality of gaze fluctuations may support an extension of the multifractal support for QE. The present results do not include gaze data, but we hope it is informative, especially in support of the postural-kinematic hypothesis, that the QE instructions enlist the nonlinear interactions across scales of the movement system without drawing the athlete's attention to the body.

Ultimately, this task- and anatomically-specific portrait of multifractal fluctuations rests on two even more generic facts. First, multifractal fluctuations spread across the body, and second, estimates of this spread predict the accuracy of the perceptual outcomes (Mangalam, Carver, et al., 2020a, 2020b). Hence, rather than inventorying body parts and task constraints prompting different values of multifractality, the longer view of investigating visually-guided aiming must examine the flow of multifractal fluctuations. Carver et al. (2017) found that multifractality spread from hip to head, as well as from head and hip toward the throwing hand. Indeed, in all these studies, the body appears to direct multifractal fluctuations towards those body parts most clearly engaged in the focal tasks. We might expect similar results in golf putting. QE training may promote this spread by reducing intentional movements of the head and eyes that might interfere with upstream flows of multifractality. The direction of attention towards a distant target in the visual field may depend on stabilizing the postural grasp of the surface underfoot and, perhaps, shunting multifractal fluctuations toward the head and eyes. Indeed, executive functions directing attention to specific features of the visual field benefits from a rich substrate of multifractal fluctuations in the head (Kelty-Stephen \& Dixon, 2014), hand (Anastas et al., 2014; Stephen et al., 2012), and brain (Kardan, Adam, et al., 2020; Kardan, Layden, et al., 2020) that vary systematically with access to and mastery of the rules supporting task performance. When we consider that eye movements show multifractality (Kelty-Stephen \& Mirman, 2013) and that variability in the monofractal structure of eye movements can predict visual attention (Wallot et al., 2015), we see early glimpses of a multifractal bridge from the postural-kinematic support to the visual support for QE.

The novelty of multifractal modeling to behavioral sciences can make this promise of a multifractal infrastructure supporting visually guided behaviors sound too ethereal and misty to be practical. But we can root this proposal in the known physiology spanning the very same bones, muscles, bones, joints, brain, and eyes whose coordination generates the winning golf putt. Specifically, the connective tissues composing the fascia operate to coordinate context-sensitive behavior across so wide a variety of scales (e.g. (Ingber, 2006)) that multifractality is one of the few current compelling frameworks for modeling how it might support perception-action (Schleip et al., 2014; Turvey \& Fonseca, 2014). The flow of multifractal fluctuations through the body could inform future explanations of how QE training and other visual aiming tasks depend on this bodywide network of connective tissues. 


\section{References}

Allison, P. D. (1977). Testing for interaction in multiple regression. American Journal of Sociology, 83(1), 144-153. https://doi.org/10.1086/226510

Anastas, J. R., Kelty-Stephen, D. G., \& Dixon, J. A. (2014). Executive function as an interactiondominant process. Ecological Psychology, 26(4), 282. https://doi.org/10.1080/10407413.2014.957985

Ashkenazy, Y., M. Hausdorff, J., Ch. Ivanov, P., \& Eugene Stanley, H. (2002). A stochastic model of human gait dynamics. Physica A: Statistical Mechanics and Its Applications, 316(1), 662-670. https://doi.org/10.1016/S0378-4371(02)01453-X

Balasubramaniam, R., Riley, M. A., \& Turvey, M. T. (2000). Specificity of postural sway to the demands of a precision task. Gait \& Posture, 11(1), 12-24. https://doi.org/10.1016/S09666362(99)00051-X

Barlow, H. B. (1963). Slippage of contact lenses and other artefacts in relation to fading and regeneration of supposedly stable retinal images. Quarterly Journal of Experimental Psychology, 15(1), 36-51. https://doi.org/10.1080/17470216308416550

Bates, D., Sarkar, D., Bates, M., \& Matrix, L. (2007). The lme4 package (R package version 2).

Bell, C., Carver, N., Zbaracki, J., \& Kelty-Stephen, D. (2019). Nonlinear amplification of variability through interaction across scales supports greater accuracy in manual aiming: Evidence from a multifractal analysis with comparisons to linear surrogates in the Fitts task. Frontiers in Physiology, 10, 998. https://doi.org/10.3389/fphys.2019.00998

Bernstein, N. A. (1967). The Co-ordination and Regulation of Movement. Pergamon Press.

Booth, C. R., Brown, H. L., Eason, E. G., Wallot, S., \& Kelty-Stephen, D. G. (2018). Expectations on hierarchical scales of discourse: Multifractality predicts both short- and long-range effects of violating gender expectations in text reading. Discourse Processes, 55(1), 12-30. https://doi.org/10.1080/0163853X.2016.1197811

Burton, G., \& Turvey, M. T. (1990). Perceiving the lengths of rods that are held but not wielded. Ecological Psychology, 2(4), 295-324. https://doi.org/10.1207/s15326969eco0204_1

Carello, C., Fitzpatrick, P., Domaniewicz, I., Chan, T.-C., \& Turvey, M. T. (1992). Effortful touch with minimal movement. Journal of Experimental Psychology: Human Perception and Performance, 18(1), 290-302. https://doi.org/10.1037/0096-1523.18.1.290

Carver, N. S., Bojovic, D., \& Kelty-Stephen, D. G. (2017). Multifractal foundations of visually-guided aiming and adaptation to prismatic perturbation. Human Movement Science, 55, 61-72. https://doi.org/10.1016/j.humov.2017.07.005 
762

763

764

765

766

767

768

769

770

771

772

773

774

775

776

777

778

779

780

781

782

783

784

785

786

787

788

789

790

791

792

793

794

795

796

Chen, L.-C., Metcalfe, J. S., Chang, T.-Y., Jeka, J. J., \& Clark, J. E. (2008). The development of infant upright posture: Sway less or sway differently? Experimental Brain Research, 186(2), 293-303. https://doi.org/10.1007/s00221-007-1236-1

Chhabra, A., \& Jensen, R. V. (1989). Direct determination of the $f(\alpha)$ singularity spectrum. Physical Review Letters, 62(12), 1327-1330. https://doi.org/10.1103/PhysRevLett.62.1327

Cooke, A., Kavussanu, M., McIntyre, D., \& Ring, C. (2010). Psychological, muscular and kinematic factors mediate performance under pressure. Psychophysiology, 47(6), 1109-1118. https://doi.org/10.1111/j.1469-8986.2010.01021.x

Coppola, D., \& Purves, D. (1996). The extraordinarily rapid disappearance of entopic images. Proceedings of the National Academy of Sciences, 93(15), 8001-8004. https://doi.org/10.1073/pnas.93.15.8001

Davids, K., \& Araújo, D. (2016). What could an ecological dynamics rationale offer Quiet Eye research? Comment on Vickers. Current Issues in Sport Science (CISS), 1, 104. https://doi.org/10.15203/CISS_2016.100

de Oliveira, R. F. (2016). Visual perception in expert action. In M Raab, B. Lobinger, S. Hoffman, A. Pizzera, \& S. Laborde (Eds.), Performance Psychology: Perception, Aaction, Cognition, Emotion (pp. 254-270). Academic Press.

Diniz, A., Wijnants, M. L., Torre, K., Barreiros, J., Crato, N., Bosman, A. M. T., Hasselman, F., Cox, R. F. A., Van Orden, G. C., \& Delignières, D. (2011). Contemporary theories of $1 / f$ noise in motor control. Human Movement Science, 30(5), 889-905. https://doi.org/10.1016/j.humov.2010.07.006

Ditchburn, R. W., \& Ginsborg, B. L. (1952). Vision with a stabilized retinal image. Nature, 170(4314), 36-37. https://doi.org/10.1038/170036a0

Dixon, J. A., Holden, J. G., Mirman, D., \& Stephen, D. G. (2012). Multifractal dynamics in the emergence of cognitive structure. Topics in Cognitive Science, 4(1), 51-62. https://doi.org/10.1111/j.1756-8765.2011.01162.x

Doyon, J. K., Hajnal, A., Surber, T., Clark, J. D., \& Kelty-Stephen, D. G. (2019). Multifractality of posture modulates multisensory perception of stand-on-ability. PLOS ONE, 14(2), e0212220. https://doi.org/10.1371/journal.pone.0212220

Feldman, A. G. (2018). Indirect, referent control of motor actions underlies directional tuning of neurons. Journal of Neurophysiology, 121(3), 823-841. https://doi.org/10.1152/jn.00575.2018

Feldman, A. G., \& Zhang, L. (2020). Eye and head movements and vestibulo-ocular reflex in the context of indirect, referent control of motor actions. Journal of Neurophysiology, 124(1), 115133. https://doi.org/10.1152/jn.00076.2020

Frenz, H., Bremmer, F., \& Lappe, M. (2003). Discrimination of travel distances from 'situated' optic flow. Vision Research, 43(20), 2173-2183. https://doi.org/10.1016/S0042-6989(03)00337-7 
797

803

804

805

806

807

808

809

810

811

812

813

814

815

816

817

818

819

820

821

822

823

824

825

826

827

828

829

830

831

832

Friston, K., Breakspear, M., \& Deco, G. (2012). Perception and self-organized instability. Frontiers in Computational Neuroscience, 6, 44. https://doi.org/10.3389/fncom.2012.00044

Gallicchio, G., Cooke, A., \& Ring, C. (2016). Lower left temporal-frontal connectivity characterizes expert and accurate performance: High-alpha T7-Fz connectivity as a marker of conscious processing during movement. Sport, Exercise, and Performance Psychology, 5(1), 14-24. https://doi.org/10.1037/spy0000055

Gallicchio, G., Cooke, A., \& Ring, C. (2017). Practice makes efficient: Cortical alpha oscillations are associated with improved golf putting performance. Sport, Exercise, and Performance Psychology, 6(1), 89-102. https://doi.org/10.1037/spy0000077

Gallicchio, G., \& Ring, C. (2020). The quiet eye effect: A test of the visual and postural-kinematic hypotheses. Sport, Exercise, and Performance Psychology, 9(1), 143-159. https://doi.org/10.1037/spy0000162

Gibson, J. J. (1979). The Ecological Approach to Visual Perception. Houghton Mifflin.

Hajnal, A., Bunch, D. A., \& Kelty-Stephen, D. G. (2014). Going for distance and going for speed: Effort and optical variables shape information for distance perception from observation to response. Attention, Perception, \& Psychophysics, 76(4), 1015-1035. https://doi.org/10.3758/s13414-014-0629-z

Hajnal, A., Bunch, D. A., \& Kelty-Stephen, D. G. (2016). Pulling out all the stops to make the distance: Effects of effort and optical information in distance perception responses made by rope pulling. Attention, Perception, \& Psychophysics, 78(2), 685-699. https://doi.org/10.3758/s13414015-1035-X

Hajnal, A., Clark, J. D., Doyon, J. K., \& Kelty-Stephen, D. G. (2018). Fractality of body movements predicts perception of affordances: Evidence from stand-on-ability judgments about slopes. Journal of Experimental Psychology: Human Perception and Performance, 44(6), 836-841. https://doi.org/10.1037/xhp0000510

Haken, H., Kelso, J. A. S., \& Bunz, H. (1985). A theoretical model of phase transitions in human hand movements. Biological Cybernetics, 51(5), 347-356. https://doi.org/10.1007/BF00336922

Harrison, S. J., \& Stergiou, N. (2015). Complex adaptive behavior and dexterous action. Nonlinear Dynamics, Psychology, and Life Sciences, 19(4), 345-394. https://pubmed.ncbi.nlm.nih.gov/26375932

Holt, K. G., Wagenaar, R. O., \& Saltzman, E. (2010). A dynamic systems: Constraints approach to rehabilitation. Brazilian Journal of Physical Therapy, 14, 446-463. https://doi.org/10.1590/S1413-35552010000600002

Houdijk, H., Brown, S. E., \& van Dieën, J. H. (2015). Relation between postural sway magnitude and metabolic energy cost during upright standing on a compliant surface. Journal of Applied Physiology, 119(6), 696-703. https://doi.org/10.1152/japplphysiol.00907.2014 
833

834

835

836

837

838

839

840

841

842

843

844

845

846

847

848

849

850

851

852

853

854

855

856

857

858

859

860

861

862

863

864

865

866

867

Ihlen, E. A. F., \& Vereijken, B. (2010). Interaction-dominant dynamics in human cognition: Beyond 1/f fluctuation. Journal of Experimental Psychology: General, 139(3), 436-463. https://doi.org/10.1037/a0019098

Ingber, D. E. (2006). Cellular mechanotransduction: Putting all the pieces together again. The FASEB Journal, 20(7), 811-827. https://doi.org/10.1096/fj.05-5424rev

Ivanov, P. C., Nunes Amaral, L. A., Goldberger, A. L., Havlin, S., Rosenblum, M. G., Stanley, H. E., \& Struzik, Z. R. (2001). From 1/f noise to multifractal cascades in heartbeat dynamics. Chaos: An Interdisciplinary Journal of Nonlinear Science, 11(3), 641-652. https://doi.org/10.1063/1.1395631

Kardan, O., Adam, K. C. S., Mance, I., Churchill, N. W., Vogel, E. K., \& Berman, M. G. (2020). Distinguishing cognitive effort and working memory load using scale-invariance and alpha suppression in EEG. Neurolmage, 211,

116622. https://doi.org/10.1016/j.neuroimage.2020.116622

Kardan, O., Layden, E., Choe, K. W., Lyu, M., Zhang, X., Beilock, S. L., Rosenberg, M. D., \& Berman, M. G. (2020). Scale-invariance in brain activity predicts practice effects in cognitive performance. BioRxiv, 114959. https://doi.org/10.1101/2020.05.25.114959

Kello, C. T. (2018). Editor's introduction and review: Coordination and context in cognitive science. Topics in Cognitive Science, 10(1), 6-17. https://doi.org/10.1111/tops.12302

Kelty-Stephen, D. G. (2018). Multifractal evidence of nonlinear interactions stabilizing posture for phasmids in windy conditions: A reanalysis of insect postural-sway data. PLOS ONE, 13(8), e0202367. https://doi.org/10.1371/journal.pone.0202367

Kelty-Stephen, D. G., \& Dixon, J. A. (2014). Interwoven fluctuations during intermodal perception: Fractality in head sway supports the use of visual feedback in haptic perceptual judgments by manual wielding. Journal of Experimental Psychology: Human Perception and Performance, 40(6), 2289-2309. https://doi.org/10.1037/a0038159

Kelty-Stephen, D. G., Lee, I.-C., Carver, N. S., Newell, K. M., \& Mangalam, M. (2020). Visual effort moderates a self-correcting nonlinear postural control policy. BioRxiv, 209502. https://doi.org/10.1101/2020.07.17.209502

Kelty-Stephen, D. G., \& Mirman, D. (2013). Gaze fluctuations are not additively decomposable: Reply to Bogartz and Staub. Cognition, 126(1), 128-134. https://doi.org/10.1016/j.cognition.2012.09.002

Kelty-Stephen, D. G., Stirling, L. A., \& Lipsitz, L. A. (2016). Multifractal temporal correlations in circle-tracing behaviors are associated with the executive function of rule-switching assessed by the Trail Making Test. Psychological Assessment, 28(2), 171-180. https://doi.org/10.1037/pas0000177 
868

869

870

871

872

873

874

875

876

877

878

879

880

881

882

883

884

885

886

887

888

889

890

891

892

893

894

895

896

897

898

899

900

901

902

Kelty-Stephen, D. G., \& Wallot, S. (2017). Multifractality versus (mono-) fractality as evidence of nonlinear interactions across timescales: Disentangling the belief in nonlinearity from the diagnosis of nonlinearity in empirical data. Ecological Psychology, 29(4), 259-299. https://doi.org/10.1080/10407413.2017.1368355

Koslucher, F., Munafo, J., \& Stoffregen, T. A. (2016). Postural sway in men and women during nauseogenic motion of the illuminated environment. Experimental Brain Research, 234(9), 27092720. https://doi.org/10.1007/s00221-016-4675-8

Krueger, E., Schneider, A., Chavaillaz, A., Groner, R., Sawyer, B. D., Sonderegger, A., \& Hancock, P. A. (2019). Microsaccades distinguish looking from seeing. Journal of Eye Movement Research, $12(6), 2$.

Latash, M. L. (2012). The bliss (not the problem) of motor abundance (not redundancy). Experimental Brain Research, 217(1), 1-5. https://doi.org/10.1007/s00221-012-3000-4

Latash, M. L. (2020). On primitives in motor control. Motor Control, 24(2), 318-346. https://doi.org/10.1123/mc.2019-0099

Lederman, S. J., Ganeshan, S. R., \& Ellis, R. E. (1996). Effortful touch with minimum movement: Revisited. Journal of Experimental Psychology: Human Perception and Performance, 22(4), 851868. https://doi.org/10.1037/0096-1523.22.4.851

Lee, I.-C., Pacheco, M. M., \& Newell, K. M. (2019). The precision demands of viewing distance modulate postural coordination and control. Human Movement Science, 66, 425-439. https://doi.org/10.1016/j.humov.2019.05.019

Lee, J. T., \& Kelty-Stephen, D. G. (2017). Cascade-driven series with narrower multifractal spectra than their surrogates: Standard deviation of multipliers changes interactions across scales. Complexity, 2017, 7015243. https://doi.org/10.1155/2017/7015243

Li, R., Walter, H. J., \& Stoffregen, T. A. (2020). The role of visual feedback about motion of the ground on postural sway. Journal of Motor Behavior, 52(3), 352-359. https://doi.org/10.1080/00222895.2019.1627281

Liphart, J., Gallichio, J., Tilson, J. K., Pei, Q., Wu, S. S., \& Duncan, P. W. (2015). Concordance and discordance between measured and perceived balance and the effect on gait speed and falls following stroke. Clinical Rehabilitation, 30(3), 294-302. https://doi.org/10.1177/0269215515578294

Lipsitz, L. A. (2002). Dynamics of stability: The physiologic basis of functional health and frailty. The Journals of Gerontology: Series A, 57(3), B115-B125. https://doi.org/10.1093/gerona/57.3.B115

Lipsitz, L. A., \& Goldberger, A. L. (1992). Loss of "complexity" and aging: Potential applications of fractals and chaos theory to senescence. JAMA, 267(13), 1806-1809. https://doi.org/10.1001/jama.1992.03480130122036 
903

904

905

906

907

908

909

910

911

912

913

914

915

916

917

918

919

920

921

922

923

924

925

926

927

928

929

930

931

932

933

934

935

936

Mangalam, M., Carver, N. S., \& Kelty-Stephen, D. G. (2020a). Global broadcasting of local fractal fluctuations in a bodywide distributed system supports perception via effortful touch. Chaos, Solitons \& Fractals, 135, 109740. https://doi.org/10.1016/j.chaos.2020.109740

Mangalam, M., Carver, N. S., \& Kelty-Stephen, D. G. (2020b). Multifractal signatures of perceptual processing on anatomical sleeves of the human body. Journal of The Royal Society Interface, 17(168), 20200328. https://doi.org/10.1098/rsif.2020.0328

Mangalam, M., \& Kelty-Stephen, D. G. (2020). Multiplicative-cascade dynamics supports whole-body coordination for perception via effortful touch. Human Movement Science, 70, 102595. https://doi.org/10.1016/j.humov.2020.102595

Mangalam, M., Lee, I.-C., Newell, K. M., \& Kelty-Stephen, D. G. (2020). Visual effort moderates postural cascade dynamics. BioRxiv, 209486. https://doi.org/10.1101/2020.07.17.209486

Mark, L. S. (1987). Eyeheight-scaled information about affordances: A study of sitting and stair climbing. Journal of Experimental Psychology: Human Perception and Performance, 13(3), 361370. https://doi.org/10.1037/0096-1523.13.3.361

Mark, L. S., Balliett, J. A., Craver, K. D., Douglas, S. D., \& Fox, T. (1990). What an actor must do in order to perceive the affordance for sitting. Ecological Psychology, 2(4), 325-366. https://doi.org/10.1207/s15326969eco0204_2

Martinez-Conde, S., Macknik, S. L., Troncoso, X. G., \& Dyar, T. A. (2006). Microsaccades counteract visual fading during fixation. Neuron, 49(2), 297-305. https://doi.org/10.1016/j.neuron.2005.11.033

Meyer, M., Stiedl, O., \& Kerman, B. (2003). Discrimination by multifractal spectrum estimation of human heartbeat interval dynamics. Fractals, 11(02), 195-204. https://doi.org/10.1142/S0218348X03002063

Moore, L. J., Vine, S. J., Cooke, A., Ring, C., \& Wilson, M. R. (2012). Quiet eye training expedites motor learning and aids performance under heightened anxiety: The roles of response programming and external attention. Psychophysiology, 49(7), 1005-1015. https://doi.org/10.1111/j.1469-8986.2012.01379.x

Moore, L. J., Vine, S. J., Freeman, P., \& Wilson, M. R. (2013). Quiet eye training promotes challenge appraisals and aids performance under elevated anxiety. International Journal of Sport and Exercise Psychology, 11(2), 169-183. https://doi.org/10.1080/1612197X.2013.773688

Morales, C. J., \& Kolaczyk, E. D. (2002). Wavelet-based multifractal analysis of human balance. Annals of Biomedical Engineering, 30(4), 588-597. https://doi.org/10.1114/1.1478082

Morrison, A., McGrath, D., \& Wallace, E. S. (2016). Motor abundance and control structure in the golf swing. Human Movement Science, 46, 129-147. https://doi.org/10.1016/j.humov.2016.01.009 
Munafo, J., Curry, C., Wade, M. G., \& Stoffregen, T. A. (2016). The distance of visual targets affects the spatial magnitude and multifractal scaling of standing body sway in younger and older adults. Experimental Brain Research, 234(9), 2721-2730. https://doi.org/10.1007/s00221-016-4676-7

Muñoz-Diosdado, A. (2005). A non linear analysis of human gait time series based on multifractal analysis and cross correlations. Journal of Physics: Conference Series, 23, 87-95. https://doi.org/10.1088/1742-6596/23/1/010

Murnaghan, C. D., Carpenter, M. G., Chua, R., \& Inglis, J. T. (2016). Keeping still doesn't “make sense": Examining a role for movement variability by stabilizing the arm during a postural control task. Journal of Neurophysiology, 117(2), 846-852. https://doi.org/10.1152/jn.01150.2015

Oftadeh, R., Haghpanah, B., Vella, D., Boudaoud, A., \& Vaziri, A. (2014). Optimal fractal-like hierarchical honeycombs. Physical Review Letters, 113(10), 104301. https://doi.org/10.1103/PhysRevLett.113.104301

Oldfield, R. C. (1971). The assessment and analysis of handedness: The Edinburgh inventory. Neuropsychologia, 9(1), 97-113. https://doi.org/10.1016/0028-3932(71)90067-4

Palatinus, Z., Dixon, J. A., \& Kelty-Stephen, D. G. (2013). Fractal fluctuations in quiet standing predict the use of mechanical information for haptic perception. Annals of Biomedical Engineering, 41(8), 1625-1634. https://doi.org/10.1007/s10439-012-0706-1

Palatinus, Z., Kelty-Stephen, D. G., Kinsella-Shaw, J., Carello, C., \& Turvey, M. T. (2014). Haptic perceptual intent in quiet standing affects multifractal scaling of postural fluctuations. Journal of Experimental Psychology: Human Perception and Performance, 40(5), 1808-1818. https://doi.org/10.1037/a0037247

Panchuk, D., \& Vickers, J. N. (2006). Gaze behaviors of goaltenders under spatial-temporal constraints. Human Movement $\quad$ Science, 25(6), 733-752. https://doi.org/10.1016/j.humov.2006.07.001

Park, H., \& Turvey, M. T. (2008). Imperfect symmetry and the elementary coordination law. In A. Fuchs \& V. K. Jirsa (Eds.), Coordination: Neural, Behavioral and Social Dynamics (pp. 3-25). Springer Berlin Heidelberg. https://doi.org/10.1007/978-3-540-74479-5_1

Profeta, V. L. S., \& Turvey, M. T. (2018). Bernstein's levels of movement construction: A contemporary perspective. Human Movement Science, 57, 111-133. https://doi.org/10.1016/j.humov.2017.11.013

Raja, V., \& Anderson, M. L. (2019). Radical embodied cognitive neuroscience. Ecological Psychology, 31(3), 166-181. https://doi.org/10.1080/10407413.2019.1615213

Rajachandrakumar, R., Mann, J., Schinkel-Ivy, A., \& Mansfield, A. (2018). Exploring the relationship between stability and variability of the centre of mass and centre of pressure. Gait \& Posture, 63, 254-259. https://doi.org/10.1016/j.gaitpost.2018.05.008 
972

973

974

975

976

977

978

979

980

981

982

983

984

985

986

987

988

989

990

991

992

993

994

995

996

997

998

999

1000

1001

1002

1003

1004

1005

Renshaw, I., Davids, K., Araújo, D., Lucas, A., Roberts, W. M., Newcombe, D. J., \& Franks, B. (2019). Evaluating weaknesses of "perceptual-cognitive training" and "brain training" methods in sport: An ecological dynamics critique. Frontiers in Psychology, 9, 2468. https://www.frontiersin.org/article/10.3389/fpsyg.2018.02468

Reynolds, A. M. (2015). Liberating Lévy walk research from the shackles of optimal foraging. Physics of Life Reviews, 14, 59-83. https://doi.org/10.1016/j.plrev.2015.03.002

Rienhoff, R., Tirp, J., Strauß, B., Baker, J., \& Schorer, J. (2016). The 'quiet eye' and motor performance: A systematic review based on Newell's constraints-led model. Sports Medicine, 46(4), 589-603. https://doi.org/10.1007/s40279-015-0442-4

Schleip, R., Mechsner, F., Zorn, A., \& Klingler, W. (2014). The bodywide fascial network as a sensory organ for haptic perception. Journal of Motor Behavior, 46(3), 191-193. https://doi.org/10.1080/00222895.2014.880306

Schlesinger, M. F., \& Klafter, J. (1986). Lévy walks versus Lévy flights. In H. E. Stanley \& N. Ostrowski (Eds.), Growth and Form (Stanley, H, pp. 279-283). Martinus Nijhoff.

Schmitt, D. T., \& Ivanov, P. C. (2007). Fractal scale-invariant and nonlinear properties of cardiac dynamics remain stable with advanced age: A new mechanistic picture of cardiac control in healthy elderly. American Journal of Physiology-Regulatory, Integrative and Comparative Physiology, 293(5), R1923-R1937. https://doi.org/10.1152/ajpregu.00372.2007

Schreiber, T., \& Schmitz, A. (1996). Improved surrogate data for nonlinearity tests. Physical Review Letters, 77(4), 635-638. https://doi.org/10.1103/PhysRevLett.77.635

Serres, J. R., \& Ruffier, F. (2017). Optic flow-based collision-free strategies: From insects to robots. Arthropod Structure \& Development, 46(5), 703-717. https://doi.org/10.1016/j.asd.2017.06.003

Shannon, C. E. (1948). A mathematical theory of communication. The Bell System Technical Journal, 27(3), 379-423. https://doi.org/10.1002/j.1538-7305.1948.tb01338.x

Shimizu, Y. U., Thurner, S., \& Ehrenberger, K. (2002). Multifractal spectra as a measure of complexity in human posture. Fractals, 10(01), 103-116. https://doi.org/10.1142/S0218348X02001130

Shine, J. M., Aburn, M. J., Breakspear, M., \& Poldrack, R. A. (2018). The modulation of neural gain facilitates a transition between functional segregation and integration in the brain. ELife, 7, e31130. https://doi.org/10.7554/eLife.31130

Stephen, D., Anastas, J., \& Dixon, J. (2012). Scaling in cognitive performance reflects multiplicative multifractal cascade dynamics. Frontiers in Physiology, 3, 102. https://doi.org/10.3389/fphys.2012.00102

Stephen, D. G., \& Mirman, D. (2010). Interactions dominate the dynamics of visual cognition. Cognition, 115(1), 154-165. https://doi.org/10.1016/j.cognition.2009.12.010 
1006

1007

1008

1009

1010

1011

1012

1013

1014

1015

1016

1017

1018

1019

1020

1021

1022

1023

1024

1025

1026

1027

1028

1029

1030

1031

1032

1033

1034

1035

1036

1037

1038

1039

1040

Stoffregen, T. A. (1985). Flow structure versus retinal location in the optical control of stance. Journal of Experimental Psychology: Human Perception and Performance, 11(5), 554-565. https://doi.org/10.1037/0096-1523.11.5.554

Stoffregen, T. A. (1986). The role of optical velocity in the control of stance. Perception \& Psychophysics, 39(5), 355-360. https://doi.org/10.3758/BF03203004

Stoffregen, T. A., Mantel, B., \& Bardy, B. G. (2017). The senses considered as one perceptual system. Ecological Psychology, 29(3), 165-197. https://doi.org/10.1080/10407413.2017.1331116

Stoffregen, T. A., \& Riccio, G. E. (1990). Responses to optical looming in the retinal center and periphery. Ecological Psychology, 2(3), 251-274. https://doi.org/10.1207/s15326969eco0203_3

Stoffregen, T. A., Yang, C.-M., \& Bardy, B. G. (2005). Affordance judgments and nonlocomotor body movement. Ecological Psychology, 17(2), 75-104. https://doi.org/10.1207/s15326969eco1702_2

Teng, D. W., Eddy, C. L., \& Kelty-Stephen, D. G. (2016). Non-visually-guided distance perception depends on matching torso fluctuations between training and test. Attention, Perception, \& Psychophysics, 78(8), 2320-2328. https://doi.org/10.3758/s13414-016-1213-5

Turvey, M T. (1977). Contrasting orientations to the theory of visual information processing. Psychological Review, 84(1), 67-88. https://doi.org/10.1037/0033-295X.84.1.67

Turvey, Michael T, \& Fonseca, S. T. (2014). The medium of haptic perception: A tensegrity hypothesis. Journal of Motor Behavior, 46(3), 143-187. https://doi.org/10.1080/00222895.2013.798252

van Smeden, M., de Groot, J. A. H., Moons, K. G. M., Collins, G. S., Altman, D. G., Eijkemans, M. J. C., \& Reitsma, J. B. (2016). No rationale for 1 variable per 10 events criterion for binary logistic regression analysis. BMC Medical Research Methodology, 16(1), 163. https://doi.org/10.1186/s12874-016-0267-3

Vaz, D. V., Silva, P. L., Mancini, M. C., Carello, C., \& Kinsella-Shaw, J. (2017). Towards an ecologically grounded functional practice in rehabilitation. Human Movement Science, 52, 117132. https://doi.org/10.1016/j.humov.2017.01.010

Vecera, S. P., Cosman, J. D., Vatterott, D. B., \& Roper, Z. J. J. (2014). The control of visual attention: Toward a unified account. In B. H. Ross (Ed.), The Psychology of Learning and Motivation (pp. 303-347). Academic Press.

Vickers, J. N. (1992). Gaze control in putting. Perception, 21(1), 117-132. https://doi.org/10.1068/p210117

Vickers, J. N. (1996). Visual control when aiming at a far target. Journal of Experimental Psychology: Human Perception and Performance, 22(2), 342-354. https://doi.org/10.1037/0096-1523.22.2.342

Vickers, J. N. (2009). Advances in coupling perception and action: the quiet eye as a bidirectional link between gaze, attention, and action. In Markus Raab, J. G. Johnson, \& H. R. B. T.-P. in B. R. 
Heekeren (Eds.), Mind and Motion: The Bidirectional Link between Thought and Action (Vol. 174, pp. 279-288). Elsevier. https://doi.org/10.1016/S0079-6123(09)01322-3

Vickers, J. N. (2011). Mind over muscle: The role of gaze control, spatial cognition, and the quiet eye in motor expertise. Cognitive Processing, 12(3), 219-222. https://doi.org/10.1007/s10339-0110411-2

1047

1048

Vine, S. J., Lee, D. H., Walters-Symons, R., \& Wilson, M. R. (2017). An occlusion paradigm to assess the importance of the timing of the quiet eye fixation. European Journal of Sport Science, 17(1), 85-92. https://doi.org/10.1080/17461391.2015.1073363

Vine, S. J., Lee, D. O. N., Moore, L. E. E. J., \& Wilson, M. R. (2013). Quiet eye and choking: Online control breaks down at the point of performance failure. Medicine \& Science in Sports \& Exercise, 45(10), 1988-1994. https://doi.org/10.1249/MSS.0b013e31829406c7

Vine, S., Moore, L., \& Wilson, M. (2011). Quiet eye training facilitates competitive putting performance in elite golfers. Frontiers in Psychology, 2, 8. https://doi.org/0.3389/fpsyg.2011.00008

Wagenmakers, E.-J., van der Maas, H. L. J., \& Farrell, S. (2012). Abstract concepts require concrete models: Why cognitive scientists have not yet embraced nonlinearly coupled, dynamical, selforganized critical, synergistic, scale-free, exquisitely context-sensitive, interaction-dominant, multifractal, interdependent b. Topics in Cognitive Science, 4(1), 87-93. https://doi.org/10.1111/j.1756-8765.2011.01164.x

Wallot, S., O'Brien, B., Coey, C. A., \& Kelty-Stephen, D. (2015). Power-law fluctuations in eye movements predict text comprehension during connected text reading. In D. C. Noelle, R. Dale, A. S. Warlaumont, J. Yoshimi, T. Matlock, \& C. D. Jennings (Eds.), Proceedings of the 37th Annual Meeting of the Cognitive Science Society (pp. 2583-2588).

Warren, W. H. (1984). Perceiving affordances: Visual guidance of stair climbing. Journal of Experimental Psychology: Human Perception and Performance, 10(5), 683-703. https://doi.org/10.1037/0096-1523.10.5.683

West, B. J., \& Scafetta, N. (2003). Nonlinear dynamical model of human gait. Physical Review E, 67(5), 51917. https://doi.org/10.1103/PhysRevE.67.051917

Williams, A. M. (2016). Quiet eye vs. noisy brain: The eye like the brain is always active-comment on Vickers. Current Issues in Sport Science (CISS), 1, 116. https://doi.org/10.15203/CISS_2016.116

Witt, J. K., \& Proffitt, D. R. (2008). Action-specific influences on distance perception: A role for motor simulation. Journal of Experimental Psychology: Human Perception and Performance, 34(6), 1479-1492. https://doi.org/10.1037/a0010781

Table 1. Training instructions given to the quiet-eye and technical groups during the training phase. 
assume your stance, and ensure your gaze is located on the back of the ball

after setting up over the ball, fix your gaze on the hole

make no more than three fixations towards the hole

your final fixation should be a quiet eye on the back of the ball. The onset of the quiet eye should occur before the stroke begins and last for 2 to $3 \mathrm{~s}$

ensure you direct gaze towards the clubhead during the putting stroke

the quiet eye should remain on the green for 200 to $300 \mathrm{~ms}$ after the club contacts the ball take your stance with your legs shoulder-width apart

set your position so that your head is directly above the ball looking down

keep your clubhead square to the ball

allow your arms and shoulders to remain loose

the putting action should be pendulum-like, making sure that you accelerate through the ball

after contact, follow through but keep your head still and facing down

1075 Source: Adapted from (Moore et al., 2013). 
1076 Table 2. Subset of Poisson regression model including main and interaction effects non-multifractal 1077 and multifractal measures of sway, perturbation of eye height, and time

\begin{tabular}{|c|c|c|c|}
\hline predictor & $b$ & s.e.m. & $p^{*}$ \\
\hline \multicolumn{4}{|l|}{$\begin{array}{l}\text { effects of non-multifractal measures of sway } \\
\text { (see Section 3.1.1.) }\end{array}$} \\
\hline$M S D_{\text {acc }}$ & $-8.80 \times 10^{0}$ & $1.67 \times 10^{-1}$ & $<0.0001$ \\
\hline$M e a n_{\text {disp }}$ & $-2.91 \times 10^{2}$ & $1.33 \times 10^{1}$ & $<0.0001$ \\
\hline$S D_{\text {disp }}$ & $4.43 \times 10^{0}$ & $1.64 \times 10^{0}$ & $<0.01$ \\
\hline$M S D_{\mathrm{acc}} \times M e a n_{\mathrm{disp}}$ & $1.97 \times 10^{4}$ & $1.00 \times 10^{1}$ & $<0.0001$ \\
\hline$M S D_{\text {acc }} \times S D_{\text {disp }}$ & $-3.11 \times$ & $1.03 \times 10^{1}$ & $<0.0001$ \\
\hline$M e a n_{\text {disp }} \times S D_{\text {disp }}$ & $2.56 \times 10^{4}$ & $1.05 \times 10^{1}$ & $<0.0001$ \\
\hline$M S D_{\mathrm{acc}} \times M e a n_{\mathrm{disp}} \times S D_{\mathrm{disp}}$ & $-1.04 \times 10^{5}$ & $1.08 \times 10^{1}$ & $<0.0001$ \\
\hline \multicolumn{4}{|c|}{$\begin{array}{l}\text { effects of multifractal measures of sway and interactions with time } \\
\text { (see Section 3.1.2.) }\end{array}$} \\
\hline$W_{\mathrm{MF}}$ & $1.80 \times 10^{0}$ & $1.29 \times 10^{-1}$ & $<0.0001$ \\
\hline$t_{M F}$ & & & \\
\hline$W_{\mathrm{MF}}$ & -5. & 8.27 & 0.48 \\
\hline$t_{\mathrm{MF}} \times$ & 1.36 & 9.82 & 0.17 \\
\hline$t_{\mathrm{MF}} \times$ & $-8.93 \times 10^{-2}$ & $1.15 \times 10^{-2}$ & $<0.00$ \\
\hline$t_{\mathrm{MF}} \times$ trial $_{\text {block }}($ Cubic $)$ & $-1.08 \times 10^{-2}$ & $1.14 \times 10^{-2}$ & 0.34 \\
\hline \multicolumn{4}{|c|}{$\begin{array}{l}\text { interactions of multifractal measures of sway with perturbation } \\
\text { (see Section 3.1.3.) }\end{array}$} \\
\hline $\mathrm{QE} \times W_{\mathrm{MF}}$ & $-1.04 \times 10^{-0}$ & $2.03 \times 10^{-1}$ & $<0.0001$ \\
\hline QEx & -1. & 1.30 & 0.16 \\
\hline $\mathrm{QE} \times$ & $-4.59 \times 10^{-3}$ & $1.09 \times 10^{-2}$ & 0.67 \\
\hline pertı & $-1.50 \times 10^{0}$ & $3.12 \times 10^{-1}$ & $<0.0001$ \\
\hline pert & & $2.85 \times 10^{-3}$ & \\
\hline pert & & & $<0.0001$ \\
\hline QE $\times$ & 2.12 & $10^{-1}$ & $<0.0001$ \\
\hline $\mathrm{QE} \times$ & & $<10^{-3}$ & $<0.0$ \\
\hline $\mathrm{QE} \times$ perturbation $\times W_{\mathrm{MF}} \times t_{\mathrm{MF}}$ & $-2.21 \times 10^{-1}$ & $4.97 \times 10^{-2}$ & $<0.0001$ \\
\hline \multicolumn{4}{|c|}{$\begin{array}{l}\text { interactions of multifractal measures with perturbation and time } \\
\text { (see Section 3.1.4.) }\end{array}$} \\
\hline $\mathrm{QE} \times$ trial $_{\text {block }}($ Linear $) \times t_{\mathrm{MF}}$ & $-3.73 \times 10^{-2}$ & $1.46 \times 10^{-2}$ & \\
\hline QEx & $10^{-1}$ & $<10^{-2}$ & 0001 \\
\hline $\mathrm{QE} \times$ & $1.13 \times 10^{-1}$ & $1.73 \times 10^{-2}$ & $<0.0001$ \\
\hline perturl & $3.83 \times 10^{-1}$ & $5.28 \times 10^{-2}$ & $<0.0001$ \\
\hline Quadratic) $\times t_{\mathrm{MF}}$ & $4.07 \times 10^{-1}$ & $5.20 \times 10^{-2}$ & 001 \\
\hline perturbation $\times$ tri & $5.39 \times 10^{-1}$ & $4.45 \times 10^{-2}$ & $<0.0001$ \\
\hline $\mathrm{QE} \times$ perturbation $\times \mathrm{t}$ & $-2.71 \times 10^{-1}$ & $6.11 \times 10^{-2}$ & $<0.0001$ \\
\hline $\mathrm{QE} \times$ perturbation $\times$ & $5.24 \times 10^{-1}$ & $6.14 \times 10^{-2}$ & $<0.0001$ \\
\hline$t_{\mathrm{MF}}$ & & & \\
\hline $\mathrm{QE} \times$ perturbation $\times$ trial $_{\text {block }}($ Cubic $) \times t_{\mathrm{MF}}$ & $-7.90 \times 10^{-1}$ & $5.79 \times 10^{-2}$ & $<0.0001$ \\
\hline
\end{tabular}

"boldfaced values indicate statistical significance at the alpha level of 0.05 . 
bioRxiv preprint doi: https://doi.org/10.1101/2020.08.19.258053; this version posted January 12, 2021. The copyright holder for this preprint (which was not certified by peer review) is the author/funder, who has granted bioRxiv a license to display the preprint in perpetuity. It is made available under aCC-BY-NC-ND 4.0 International license.

1079 Table 3. Logistic regression modeling of successful shots.

\begin{tabular}{llll}
\hline predictor & $\boldsymbol{b}$ & s.e.m. & $\boldsymbol{p}^{*}$ \\
\hline intercept & -2.85 & 0.48 & $<\mathbf{0 . 0 0 0 1}$ \\
QE & 0.20 & 0.30 & 0.51 \\
perturbation & -0.22 & 0.43 & 0.60 \\
QE $\times$ perturbation & -1.16 & 0.65 & 0.07 \\
block & 0.24 & 0.13 & $\mathbf{0 . 0 6}$ \\
$R M S_{\text {Acc }}$ & 4.05 & 1.34 & $<\mathbf{0 . 0 1}$ \\
$W_{\mathrm{MF}}$ & -7.25 & 2.94 & $<\mathbf{0 . 0 5}$ \\
\hline
\end{tabular}

1080 *boldfaced values indicate statistical significance at the alpha level of 0.05 . 
begin with a long series of repeated measurements

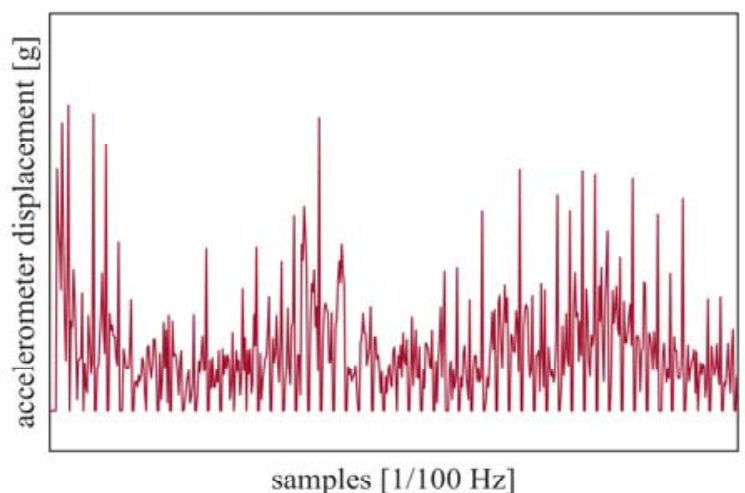

bin measurements with different window sizes, $\boldsymbol{L}$

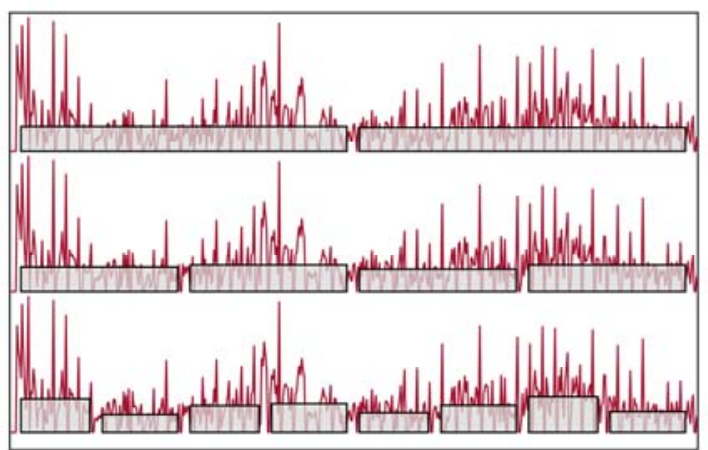

larger windows contain, on average, greater proportions of total measurements, $\boldsymbol{P}$

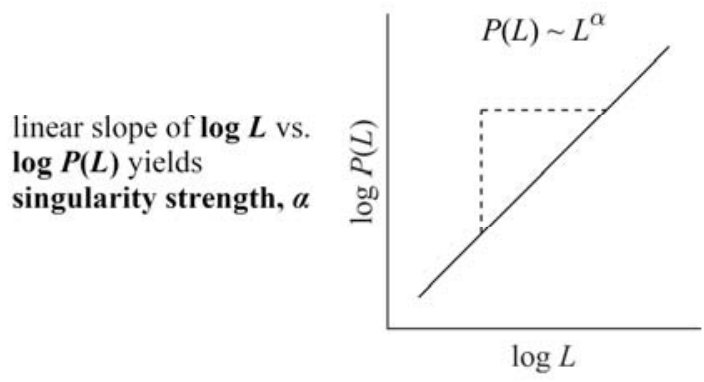

smaller windows contain greater variability in proportions of total measurements

slope from linear regression yields

\section{Hausdorff dimension, $f$}

\section{$L \boldsymbol{P}$ Entropy}

L P Entropy

L P Entropy

1081 Figure 1. Schematic of Chhabra and Jensen's (Chhabra \& Jensen, 1989) method used to estimate multifractal spectrum $f(\alpha)$ (Part-1). See Section 2.4.1. for further details. 
exponent $q$ emphasizes differently-sized measurements

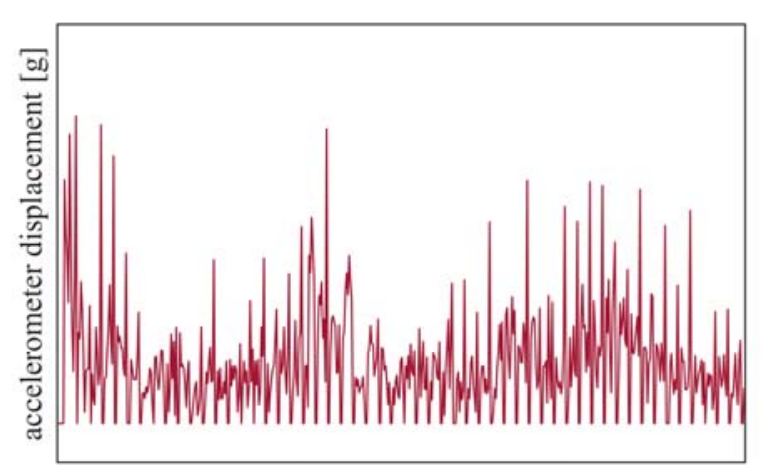

samples [1/100 Hz]

apply $\boldsymbol{q}$ to each window proportion produces mass $\boldsymbol{\mu}$

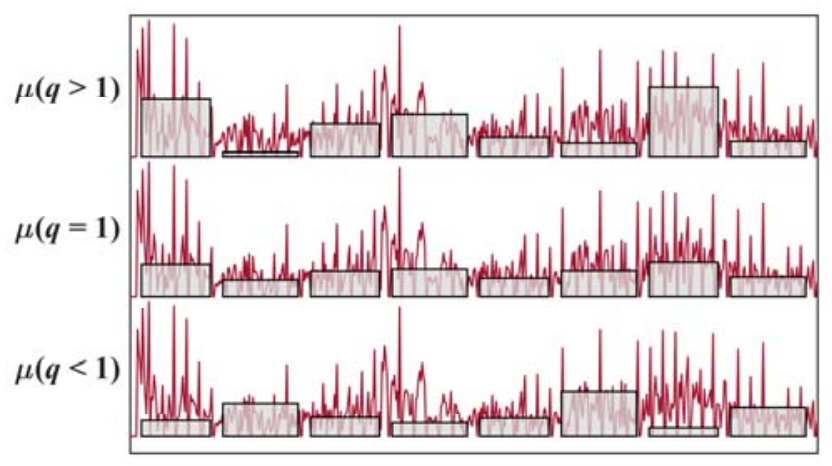

singularity strengths $\alpha(q)$ relate mass-related $\log P(L)$ to $L$

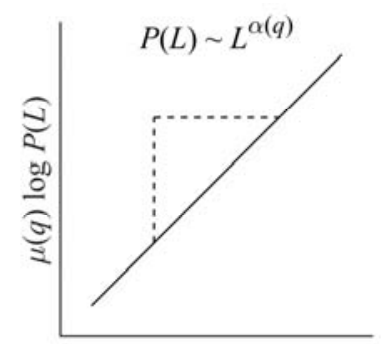

$\log L$

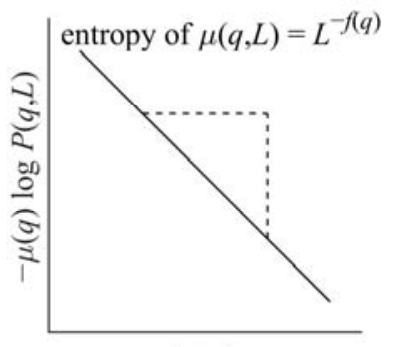

$\log L$ multifractal spectrum is the set of $\boldsymbol{\alpha}$ and $\boldsymbol{f}$ for many values of $q$

$$
q>1
$$

emphasizes large measurements

$q=1$

mass $=P$

no change

$q<1$

emphasizes small

measurements

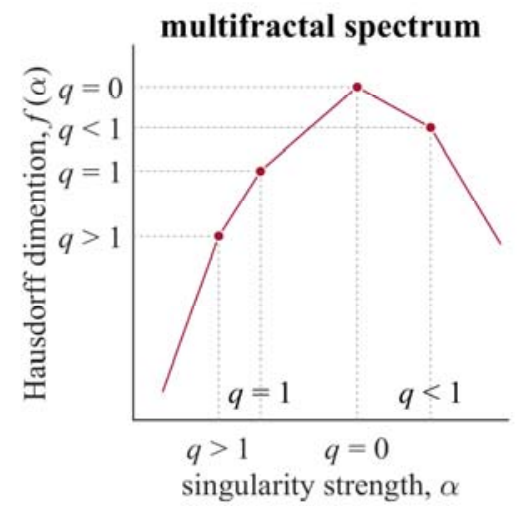

1083 Figure 2. Schematic of Chhabra and Jensen's (Chhabra \& Jensen, 1989) method used to estimate multifractal spectrum $f(\alpha)$ (Part-2). See Section 2.4.1. for further details. 
option A: multifractal spectrum width reflects strictly linear properties of the original series
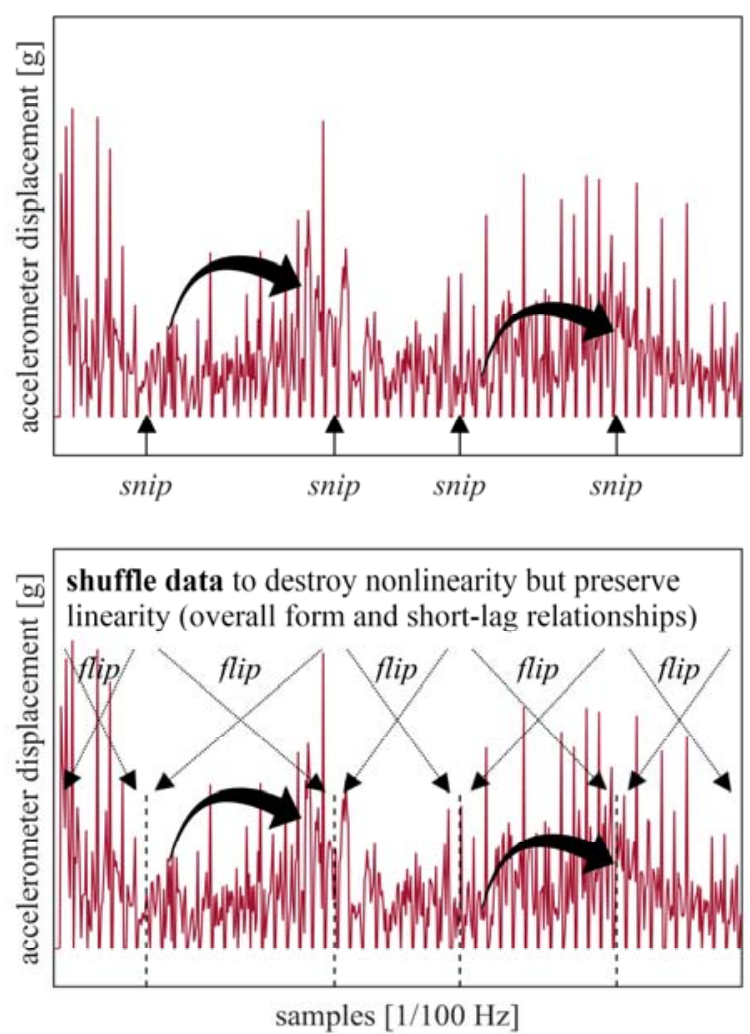

option B: multifractal spectrum width reflects effects unfloding across multiple, nested scales

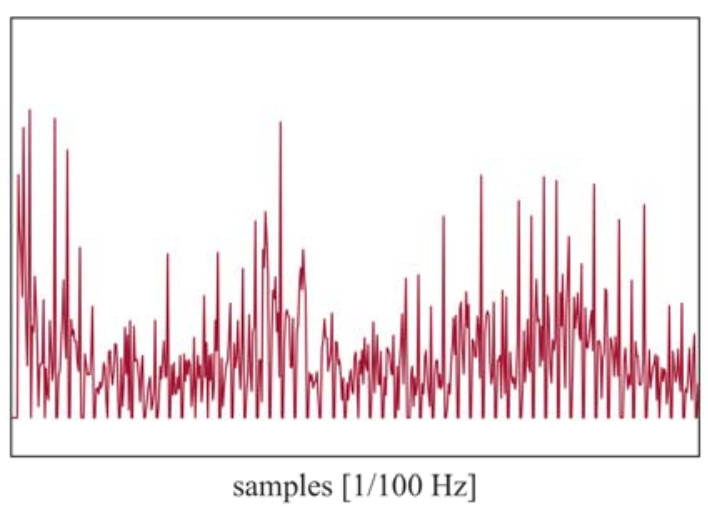

null hypothesis: multifractal spectrum width of original series does not differ from that of surrogate series

use one-sample $t$-test to compare original series' multifractal spectrum width to those of 30 surrogate series

if one-sample $t$-test is not significantly large, fail to reject null: series is linear

if one-sample $t$-test is significantly large, fail to reject null: series exhibits cascades

1085 Figure 3. Surrogate analysis used to identify whether a series exhibits multifractality due to 1086 nonlinearity (cascades). See Section 2.4.2. for further details. 

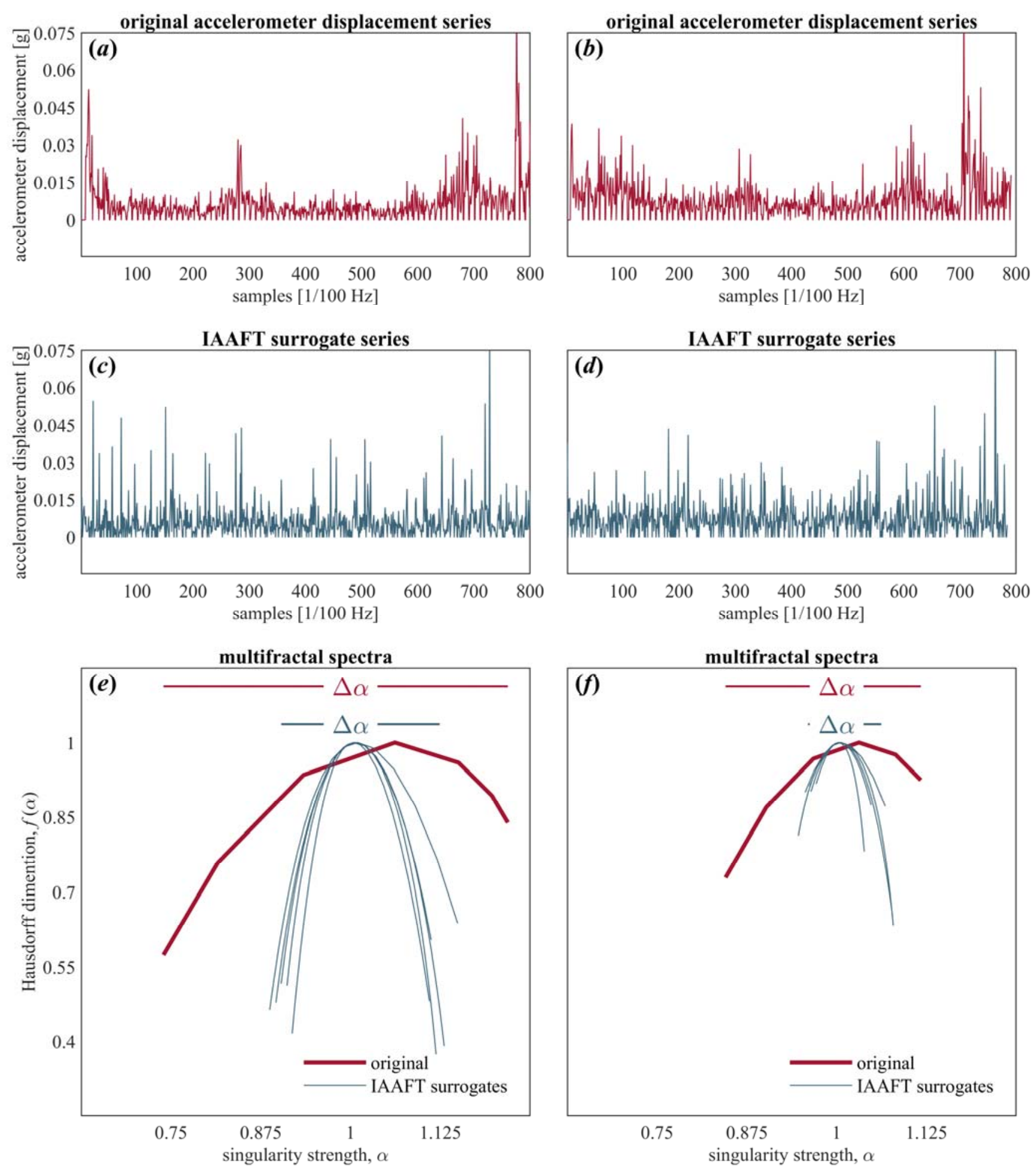

1087 Figure 4. Multifractal analysis. $(a, b)$ Representative original accelerometer displacement series. $(c, d)$ 1088 Representative IAAFT surrogate series of the original accelerometer displacement series in $a$ and $b$. (e, $1089 f$ ) Multifractal spectrum $(f(\alpha), \alpha)$ of original series in $a$ and $b$, and those of their five representative 1090 IAAFT surrogates. 

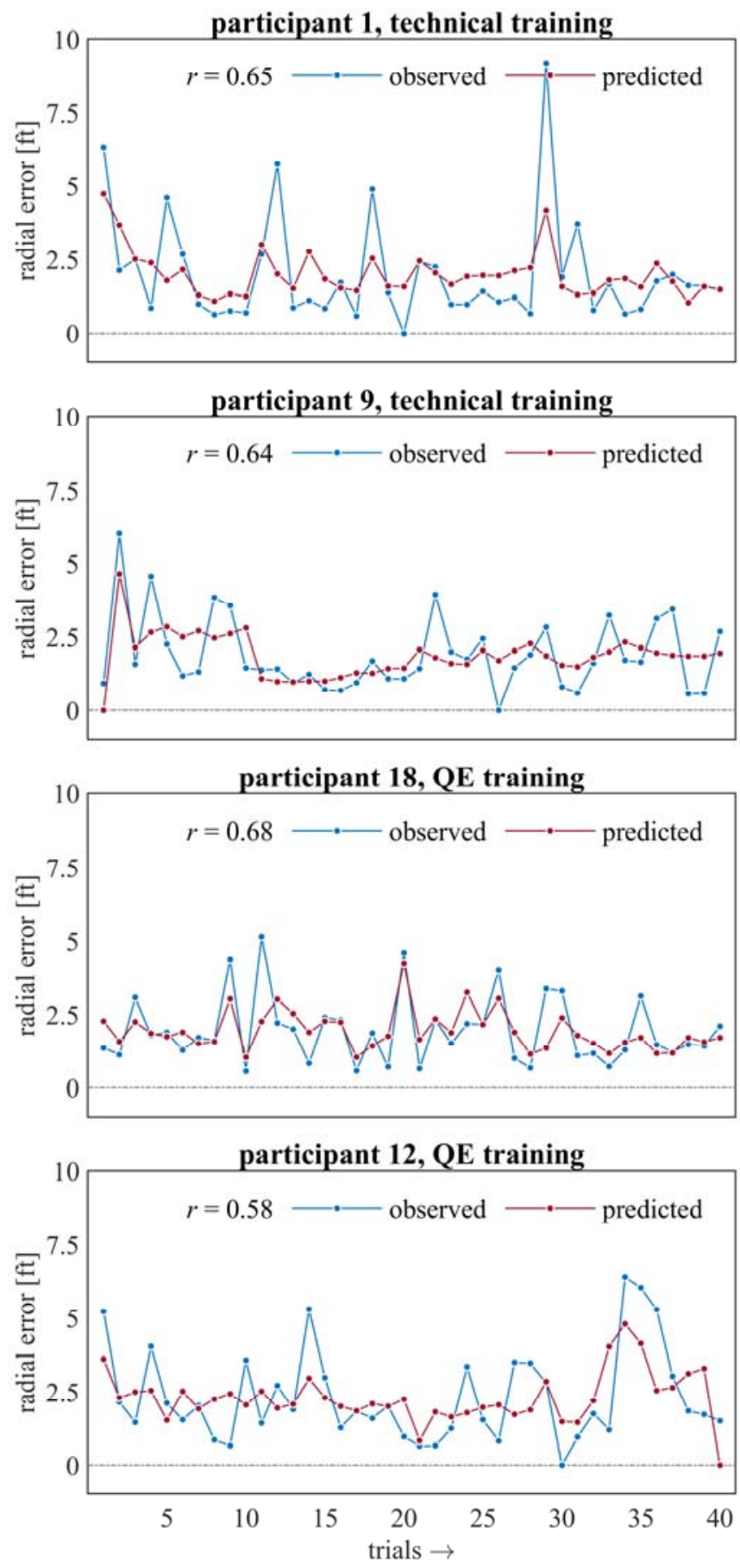

participant 15 , technical training

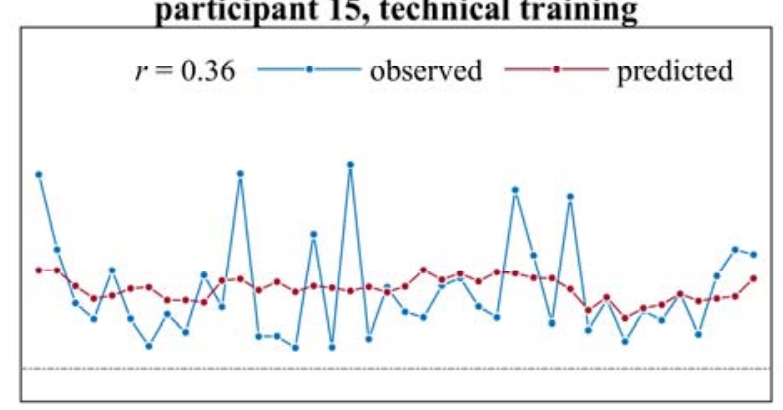

participant 3, technical training

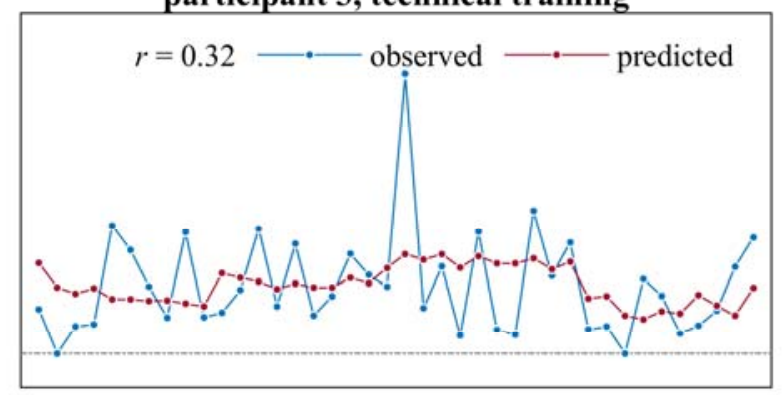

participant 14, QE training

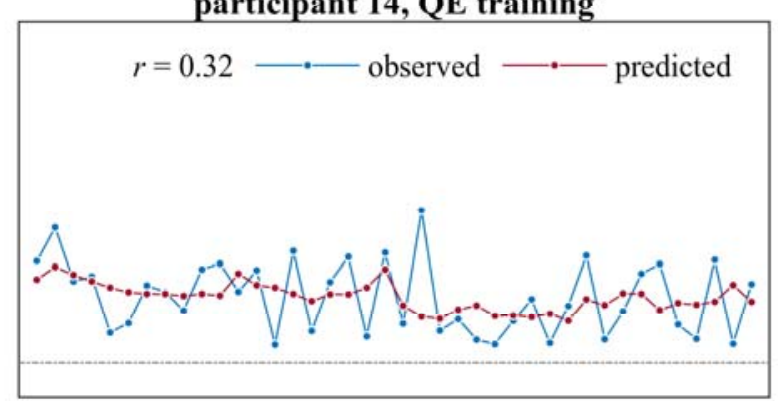

participant 20, QE training

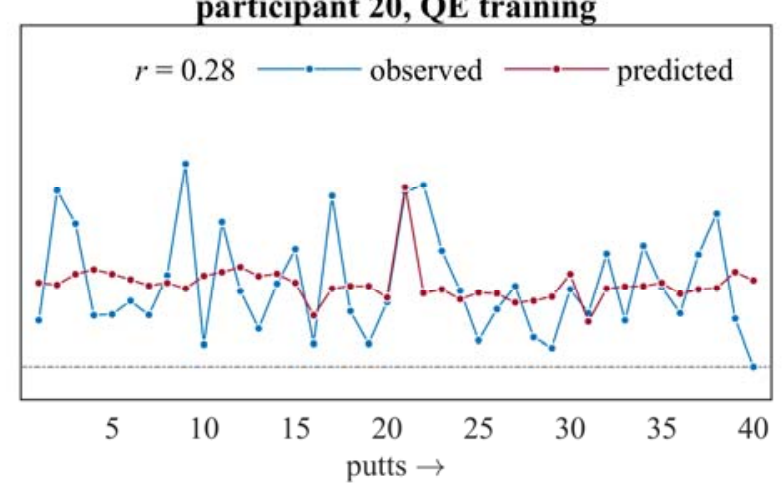

1091 Figure 5. Trial-by-trial model predictions of radial error for the two participant groups. (a) Technicaltrained group. (b) QE-trained group. 

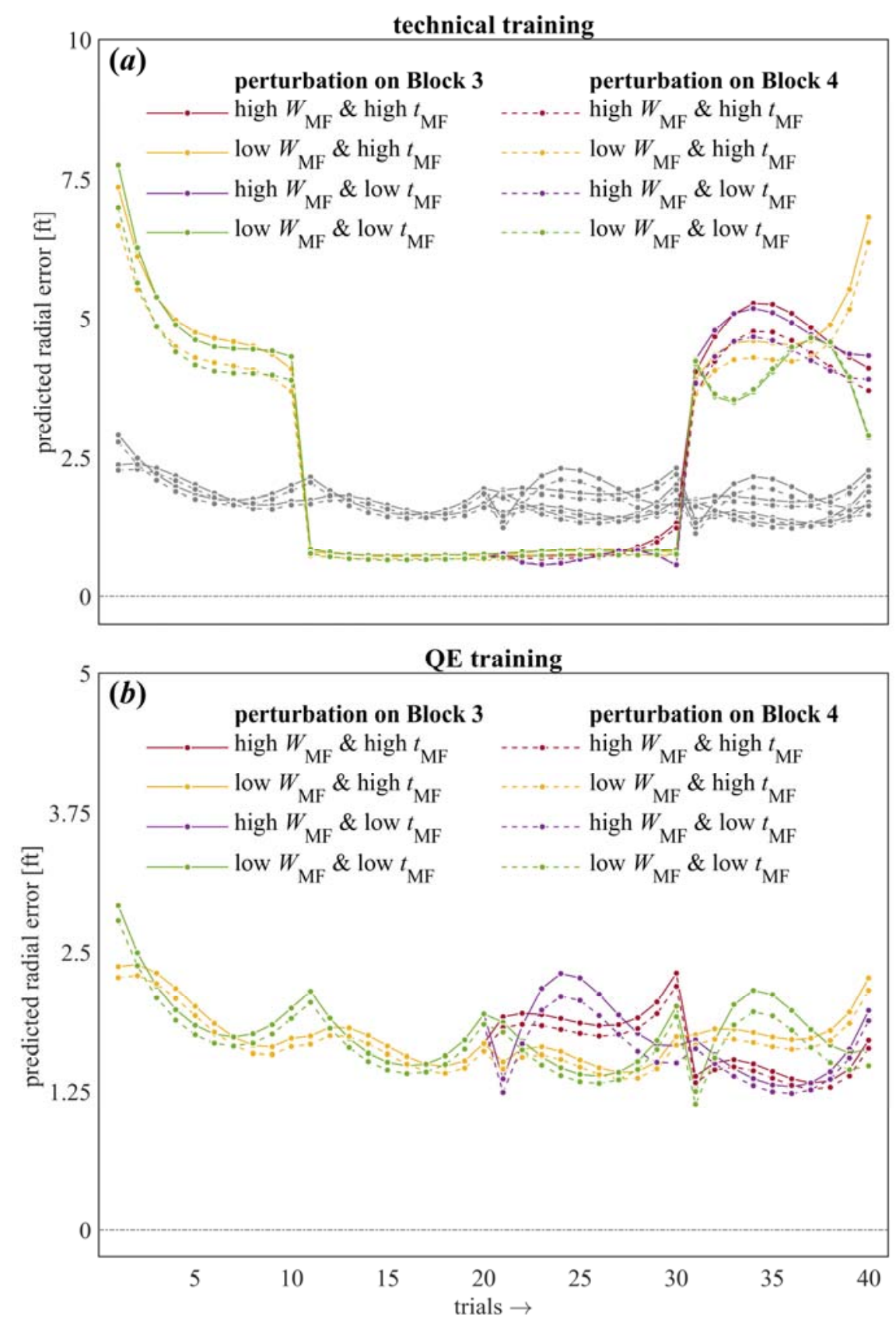

1093 Figure 6. The observed trial-by-trial radial error and trial-by-trial model predictions on the same axis 1094 for four participants each from the technical (top four panels) and QE-trained (bottom four panels) 1095 groups, respectively. The representation includes two participants each with the highest (left panels) 1096 and lowest (right panels) Pearson's correlation coefficient $r$ indicating the strength of relationship 1097 between the observed and predicted radial errors. 